University of Warwick institutional repository: http://go.warwick.ac.uk/wrap This paper is made available online in accordance with publisher policies. Please scroll down to view the document itself. Please refer to the repository record for this item and our policy information available from the repository home page for further information.

To see the final version of this paper please visit the publisher's website. Access to the published version may require a subscription.

Author(s): STEPHEN HUMPHRIES and ANTHONY MANNING

Article Title: Curves of fixed points of trace maps

Year of publication: 2007

Link to published version: http://dx.doi.org/

10.1017/S0143385707000016

Publisher statement: None 


\title{
Curves of fixed points of trace maps
}

\author{
STEPHEN HUMPHRIES $\uparrow$ and ANTHONY MANNING $\ddagger$ \\ $\dagger$ Department of Mathematics, Brigham Young University, Provo, UT 84602, USA \\ (e-mail: steve@math.byu.edu) \\ $\ddagger$ Mathematics Institute, University of Warwick, Coventry CV4 7AL, UK \\ (e-mail: A.Manning@warwick.ac.uk)
}

(Received 9 February 2006 and accepted in revised form 11 December 2006)

\begin{abstract}
We study curves of fixed points for certain diffeomorphisms of $\mathbb{R}^{3}$ that are induced by automorphisms of a trace algebra. We classify these curves. There is a function $E$ which is invariant under all such trace maps and the level surfaces $E_{t}: E=t$ are invariant; a point of $E_{t}$ will be said to have level $t$. The surface $E_{1}$ is significant. Then most fixed points on $E_{1}$ are actually on a curve $\gamma$ of fixed points interior to $E_{1}$. We describe the possibilities for the other end of $\gamma$ on $E_{1}$.
\end{abstract}

\section{Introduction}

An element $A \in P S L(2, \mathbb{Z})$ with $|\operatorname{trace}(A)|>2$ determines a hyperbolic automorphism, $\bar{A}$ say, of the 2-torus $\mathbb{T}^{2}$, which is structurally stable and has infinitely many periodic points; see $\S \S 1.8$ and 2.6 of $[\mathbf{K H}]$. Identifying a point $\left(\theta_{1}, \theta_{2}\right)^{\mathrm{T}} \in \mathbb{T}^{2}$ with $\left(-\theta_{1},-\theta_{2}\right)^{\mathrm{T}}$ gives a surface $\mathbb{T}^{2} / \sim$ homeomorphic to the 2-sphere and $\bar{A}$ induces a pseudo-Anosov diffeomorphism of this surface [FLP, CB]. This diffeomorphism is not structurally stable, and its stable and unstable foliations are not orientable. Note that (in contrast to the usual situation where the pseudo-Anosov map of a smooth surface is not differentiable at the singularities) this pseudo-Anosov map is a diffeomorphism but of a surface that is not differentiable at the singularities.

The Fricke character [FK, Go, RB1]

$$
E(x, y, z):=x^{2}+y^{2}+z^{2}-2 x y z
$$

determines level surfaces in $\mathbb{R}^{3}$ that are all smooth except for the special case of $E^{-1}(1)$, which meets the cube $[-1,1]^{3}$ in a 'curvilinear tetrahedron' that can be given the structure of $\mathbb{T}^{2} / \sim$. The braid group $B_{3}$ acts on $\mathbb{R}^{3}$ by diffeomorphisms called trace maps that preserve $E$. This action factors through $\operatorname{PSL}(2, \mathbb{Z})$, and the restriction to $E^{-1}(1) \cap[-1,1]^{3} \cong \mathbb{T}^{2} / \sim$ is given by the pseudo-Anosov diffeomorphisms described above. 
Such trace maps have been studied at higher levels $E^{-1}(t), t>1$, in $[\mathbf{C}, \mathbf{R}]$, where horseshoes appear in a way reminiscent of a 'double DA' bifurcation [W, PM p. 165, Ro p. 335]. We shall study them at the lower levels $E^{-1}(t) \cap[-1,1]^{3}, 0<t<1$; each such surface is diffeomorphic to a sphere and they each enclose the origin, $E^{-1}(0)$. By the implicit function theorem each hyperbolic fixed point of such a pseudo-Anosov diffeomorphism of our tetrahedron (except the singular points, which we call vertices) lies on a smooth curve of fixed points that descends to lower levels and may become elliptic. The diffeomorphisms of these level sets preserve a measure equivalent to the area. Thus we have a concrete situation in which to study bifurcations of area-preserving diffeomorphisms. (In contrast to the usual situation of a smooth family of diffeomorphisms of a single surface, this is a case of restricting a diffeomorphism to a smooth family of surfaces.)

Does such a curve of fixed points continue to the origin or come up again to the tetrahedron and, if so, where does it return to $E^{-1}(1)$ ? This is the question we study for a family of such maps (whose dynamics exhibit a certain symmetry) given by

$$
\left(\begin{array}{cc}
1 & n \\
-n & 1-n^{2}
\end{array}\right) \in \operatorname{PSL}(2, \mathbb{Z}), \quad n \text { even. }
$$

We shall identify these curves, which might be thought of as forming a supporting framework for the tetrahedron. That framework is present for each hyperbolic element of $\operatorname{PSL}(2, \mathbb{Z})$ but it is only at the level of the tetrahedron that the curves for different maps come together (at points with rational torus coordinates). While those fixed points have coordinates involving $\cos (2 \pi k / n)$, the expressions for the curves will involve Chebyshev polynomials.

\section{Prerequisites and statement of results}

Let $F_{2}=\left\langle x_{1}, x_{2}\right\rangle$ be a free group of rank two and let $\sigma_{i} \in \operatorname{Aut}\left(F_{2}\right), i=1,2$, be defined (as an action on the left) by

$$
\begin{array}{ll}
\sigma_{1}\left(x_{1}\right)=x_{1} x_{2}, & \sigma_{1}\left(x_{2}\right)=x_{2}, \\
\sigma_{2}\left(x_{1}\right)=x_{1}, & \sigma_{2}\left(x_{2}\right)=x_{1}^{-1} x_{2} .
\end{array}
$$

One can show that $\sigma_{1}, \sigma_{2}$ satisfy the braid relation $\sigma_{1} \sigma_{2} \sigma_{1}=\sigma_{2} \sigma_{1} \sigma_{2}$. We thus have a representation of the braid group $B_{3}$, which can be presented as $B_{3}=\left\langle\sigma_{1}, \sigma_{2}\right| \sigma_{1} \sigma_{2} \sigma_{1}=$ $\left.\sigma_{2} \sigma_{1} \sigma_{2}\right\rangle$ (see [Bi]). In fact, this representation factors through $\operatorname{PSL}(2, \mathbb{Z}) \cong B_{3} /\left\langle\left(\sigma_{1} \sigma_{2}\right)^{3}\right\rangle$. However we prefer to think of the representation as a representation of $B_{3}$ since there is a generalization of the above action to an action of the $n$-strand braid group $B_{n}$ (see [Hu2]).

Note [MKS, Theorem 3.9] that any element of $\operatorname{Aut}\left(F_{2}\right)$ fixes the conjugacy class of the commutator $x_{1} x_{2} x_{1}^{-1} x_{2}^{-1}$.

Now suppose that the $x_{i}$ are represented by elements of $S L(2, \mathbb{C})$ (a result of Epstein [E] tells us that, generically, any two such matrices generate a free group of rank two). Let

$$
t_{1}=\operatorname{trace}\left(x_{1}\right), \quad t_{2}=\operatorname{trace}\left(x_{2}\right), \quad t_{12}=\operatorname{trace}\left(x_{1} x_{2}\right) .
$$


Let $x=t_{1} / 2, y=t_{2} / 2, z=t_{12} / 2$. Recall the standard trace identities for such $2 \times 2$ matrices:

$\operatorname{trace}\left(A^{-1}\right)=\operatorname{trace}(A), \quad \operatorname{trace}\left(I_{2}\right)=2, \quad \operatorname{trace}(A B)=\operatorname{trace}(A) \operatorname{trace}(B)-\operatorname{trace}\left(A B^{-1}\right)$.

Using these we obtain the following induced action of $\sigma_{1}, \sigma_{2}$ on $\mathbb{Z}[x, y, z]$ (which we also write as an action on the left): for example, since $\sigma_{1}\left(x_{1}\right)=x_{1} x_{2}$ we have $\sigma_{1}\left(t_{1}\right)=t_{12}$ and so $\sigma_{1}(x)=z$. We similarly see that

$$
\begin{array}{lll}
\sigma_{1}(x)=z, & \sigma_{1}(y)=y, & \sigma_{1}(z)=2 y z-x \\
\sigma_{2}(x)=x, & \sigma_{2}(y)=2 x y-z, & \sigma_{2}(z)=y .
\end{array}
$$

Now because this action is obtained using the action on traces one should expect that this only guarantees an action of $B_{3}$ if we consider the action on the trace ring [Ma], this being the quotient of $\mathbb{Q}[x, y, z]$ by all generic trace relations. In terms of the generators $x, y, z$ this is the quotient of $\mathbb{Q}[x, y, z]$ by the ideal generated by the element $E-1$, the element $E-1$ being the trace of the $\operatorname{Aut}\left(F_{2}\right)$-invariant (up to conjugacy) element $x_{1} x_{2} x_{1}^{-1} x_{2}^{-1}$. However this turns out to be unnecessary as the action of $\sigma_{1}, \sigma_{2}$ on $\mathbb{Q}[x, y, z]$ is actually a representation of $B_{3}$ in $\operatorname{Aut}(\mathbb{Q}[x, y, z])$. This result is related to the fact that for any $n>1$ the braid group $B_{n}$ (see [Bi]) acts on a polynomial algebra with kernel the centre of $B_{n}$ (see [Ma, Hu1, Hu2, LPW]).

In general any automorphism or homomorphism $\phi: F_{2} \rightarrow F_{2}$ will give rise to a homomorphism of the trace ring and so determine a map $\mathbb{R}^{3} \rightarrow \mathbb{R}^{3}$. Such maps are called trace maps and have been studied by various authors [ABG, BGJ, BR, Ig, $\mathbf{L W}, \mathbf{R}, \mathbf{R B 1}, \mathbf{R B 2}, \mathbf{P}, \mathbf{P W W}, \mathbf{W W}$. For example in [RB1] the Fibonacci trace map, $(x, y, z) \mapsto(y, z, 2 y z-x)$, is studied. Information is given about curves of fixed points and period doubling.

The action (1.1) of $B_{3}$ on the polynomial ring $\mathbb{Q}[x, y, z]$ gives rise to an action of $B_{3}$ on $\mathbb{R}^{3}$ if we think of $x, y, z$ as being the usual coordinate functions for $\mathbb{R}^{3}$. We will write this action of $\alpha \in B_{3}$ on $(a, b, c)^{\mathrm{T}} \in \mathbb{R}^{3}$ on the right: $(a, b, c)^{\mathrm{T}} \alpha$; this action is also the corresponding action by Nielsen transformations [MKS, p. 130], where one uses the same conventions of left and right actions.

One checks that the action of $B_{3}$ fixes the function $E=E(x, y, z)$ of $(0.1)$ and so each level set

$$
E_{t}=\left\{(a, b, c)^{\mathrm{T}} \in \mathbb{R}^{3} \mid E(a, b, c)=t\right\}
$$

is invariant under the action. The level set $E_{1}$ is distinguished and has been drawn by various authors [Go, RB1]. There are four points

$$
V=\left\{(1,1,1)^{\mathrm{T}},(-1,-1,1)^{\mathrm{T}},(-1,1,-1)^{\mathrm{T}},(1,-1,-1)^{\mathrm{T}}\right\}
$$

in $E_{1}$ which are the only singular points of $E_{1}$. In fact, they are the only singular points of any $E_{t}, t>0$. Further, the six line segments joining these points are contained in $E_{1}$ and there is a unique component of $E_{1} \backslash V$ whose closure is compact. In fact, this closure is a topological 2-sphere that separates $\mathbb{R}^{3}$ into two components, the closure of one of these components is a 3 -ball $\mathcal{T}$ that we call a 'curvilinear tetrahedron'. One can check that $\mathcal{T} \subset[-1,1]^{3}$ and that $\mathcal{T} \cap \partial[-1,1]^{3}$ is the above-mentioned set of six line segments. 
The aim of this paper is to study the fixed points of the diffeomorphisms

$$
\alpha_{n}=\sigma_{1}^{n} \sigma_{2}^{n}: \mathbb{R}^{3} \rightarrow \mathbb{R}^{3}, \quad n>2 .
$$

We are especially interested in those fixed points which lie on $\partial \mathcal{T}$. For $n$ even these fixed points include the points $V$. If we ignore the points of $V$ for the moment, then, as pointed out in [RB1, p. 839], a consequence of the implicit function theorem is that the fixed points of $\alpha_{n}$ on $\partial \mathcal{T}$ will belong to smooth curves of fixed points. Part of what we do is to describe all of the fixed points on $\partial \mathcal{T} \backslash V$; we then describe the curves of fixed points which contain them and indicate which pairs of fixed points on $\partial \mathcal{T} \backslash V$ are joined by such smooth curves of fixed points. We say that such a pair of fixed points is $\alpha_{n}$-dual.

Let $\mathbb{T}^{2}=\mathbb{R}^{2} / \mathbb{Z}^{2}$ denote the 2-torus. If $M$ is a matrix, then $M^{\mathrm{T}}$ will denote its transpose. Define the map

$$
\Pi: \mathbb{T}^{2} \rightarrow \partial \mathcal{T}, \quad\left(\theta_{1}, \theta_{2}\right)^{\mathrm{T}} \mapsto\left(\cos \left(2 \pi \theta_{1}\right), \cos \left(2 \pi \theta_{2}\right), \cos \left(2 \pi\left(\theta_{1}+\theta_{2}\right)\right)\right)^{\mathrm{T}} .
$$

Note that $\Pi\left(\theta_{1}, \theta_{2}\right)^{\mathrm{T}}=\Pi\left(-\left(\theta_{1}, \theta_{2}\right)^{\mathrm{T}}\right)$. The map $\Pi$ is a branched double cover, branched over the four points $V^{\prime}=\left\{(0,0)^{\mathrm{T}},(0,1 / 2)^{\mathrm{T}},(1 / 2,0)^{\mathrm{T}},(1 / 2,1 / 2)^{\mathrm{T}}\right\} \subset \mathbb{T}^{2}$.

The action of $B_{3}$ on $\partial \mathcal{T}$ actually comes from an action of $B_{3}$ on $\mathbb{T}^{2}$, the action being determined by the homomorphism

$$
\Phi: B_{3} \rightarrow S L(2, \mathbb{Z}), \quad \sigma_{1} \mapsto\left(\begin{array}{ll}
1 & 1 \\
0 & 1
\end{array}\right), \quad \sigma_{2} \mapsto\left(\begin{array}{rr}
1 & 0 \\
-1 & 1
\end{array}\right) .
$$

For

$$
\alpha \in B_{3}, \quad \theta=\left(\begin{array}{l}
\theta_{1} \\
\theta_{2}
\end{array}\right) \in \mathbb{T}^{2}
$$

the maps П, $\Phi$ are related as follows (see Lemma 2.9):

$$
(\Pi \theta) \alpha=\Pi(\Phi(\alpha)(\theta)) .
$$

The condition $n>2$ guarantees that $\Phi\left(\alpha_{n}\right)$ is a hyperbolic map of the torus.

The fixed points of $\alpha$ on $\partial \mathcal{T} \backslash V$ are of two types. First note that if $\Pi(\theta) \in \partial \mathcal{T} \backslash V \subset \mathbb{R}^{3}$ is fixed by $\alpha$, then by (1.2) we must have $\Phi(\alpha)(\theta)= \pm \theta$. A fixed point $\Pi(\theta)$ is called $\alpha$-preserving, or just preserving if $\alpha$ is understood, if we have $\Phi(\alpha)(\theta)=\theta$; otherwise it is called reversing.

The fixed points on $\partial \mathcal{T}$ and the fixed curves that contain them will be put in four families:

(F1) straight line curves;

(F2) curves in the planes $x= \pm y$;

(F3) curves not meeting the planes $x= \pm y$.

For convenience we define a type (F4) curve, which will be a curve not of type (F2) but which bifurcates at points on the planes $x= \pm y$. We will initially show (in fact it should already be clear) that any fixed point on $\partial \mathcal{T} \backslash V$ belongs to a curve of fixed points of the types (F1), (F2), (F3), or (F4); however we will also show that there are no curves of fixed points of type (F4).

In fact it will follow from what we do that any fixed point for $\alpha_{n}$ is on one of the curves of the types (F1), (F2) or (F3). 
We now say a little about each of the cases (F1)-(F4), leaving most of the details and proofs to later sections.

(F1) The straight line cases. For $N \in \mathbb{N}$ let $K_{N} \subset S L(2, \mathbb{Z})$ denote the congruence $N$ subgroup of $S L(2, \mathbb{Z})$, namely the kernel of the homomorphism $S L(2, \mathbb{Z}) \rightarrow S L(2, \mathbb{Z} / N \mathbb{Z})$. Note that $\Phi\left(\alpha_{n}\right)=\Phi\left(\sigma_{1}^{n} \sigma_{2}^{n}\right) \in K_{n}$. For $k, m \in \mathbb{Z}$ and any $\beta \in B_{3}$ such that $\Phi(\beta) \in K_{n}$, it follows that any $\Pi(k / n, m / n)$ is a preserving fixed point of $\beta$. In particular, this is the case for $\alpha_{n}$.

We will show later that for most integer values of $k, m, n$ the vertical line

$$
p(z)=(\cos (2 \pi k / n), \cos (2 \pi m / n), z)^{\mathrm{T}} \subset \mathbb{R}^{3}
$$

is a line of fixed points for $\alpha_{n}$ which contains $\Pi(k / n, m / n)^{\mathrm{T}}$ and is not tangential to $\partial \mathcal{T}$ at $\Pi(k / n, m / n)^{\mathrm{T}}$. Thus this line meets $\partial \mathcal{T}$ at another point, which happens to be $\Pi(k / n,-m / n)^{\mathrm{T}}$. Thus $\Pi(k / n, m / n)^{\mathrm{T}}$ and $\Pi(k / n,-m / n)^{\mathrm{T}}$ are $\alpha_{n}$-dual.

Let $X, Y, Z \subset \mathbb{R}^{3}$ denote the $x$-axis, the $y$-axis and the $z$-axis. Now it is easily checked that any point $p \in X \cup Y \cup Z$ is fixed by each $\sigma_{i}^{4}, i=1,2$. Thus if $n$ is a multiple of 4 , then each of $X, Y, Z$ is a line of fixed points for $\alpha_{n}$ which intersects $\partial \mathcal{T}$ in $\alpha_{n}$-dual points $\left\{(1,0,0)^{\mathrm{T}},(-1,0,0)^{\mathrm{T}}\right\},\left\{(0,1,0)^{\mathrm{T}},(0,-1,0)^{\mathrm{T}}\right\},\left\{(0,0,1)^{\mathrm{T}},(0,0,-1)^{\mathrm{T}}\right\} \subset \mathbb{R}^{3}$. This concludes our discussion of the straight line cases.

(F2) The $x= \pm y$ cases. We now consider the fixed points $p=(a, b, c)^{\mathrm{T}} \in \partial \mathcal{T}$ where $a= \pm b$. The two cases are similar and so we only describe the $a=b$ case. First note that some of the straight line curves of type (F1) are in these planes. The intersection of $\mathcal{T}$ and the plane $x=y$ is a topological $\operatorname{disc} \mathcal{T}_{+}$in the $x=y$ plane bounded by the line $z=1$ and the parabola $z=2 x^{2}-1$. In this case we show that any such fixed point (if it is not on a vertical line of fixed points as in case $(\mathrm{F} 1))$ is on a curve with equation

$$
\gamma_{+}(x)=\left(x, x, x\left(1+U_{n-2}(x)\right) / U_{n-1}(x)\right)^{\mathrm{T}},
$$

where $U_{n}(x)$ is the $n$th Chebyshev polynomial.

We draw these curves as they lie in $\mathcal{T}_{+}$(bounded by $z=1$ and $z=2 x^{2}-1$ ) in Figure 1 for the case $n=20$ with $x$ horizontal and $z$ vertical; we have shown the components of the curve $\gamma_{+}(x)$ in solid and we have also indicated some solid and dashed vertical lines. The solid vertical lines are at $x$-values that are poles of the function $(1+$ $\left.U_{n-2}(x)\right) / U_{n-1}(x)$. The dashed vertical lines are lines of fixed points for $\alpha_{20}$. We have also drawn the parabola $z=x^{2}$ which indicates the points on the (dashed) vertical lines of fixed points which are where the function $E$ is minimized (see Lemma 2.8(i)).

(F3) and (F4) remaining cases. We will show that all curves of fixed points which are not completely contained in the planes $x= \pm y$ are determined by a single polynomial $K_{n}(x, y)$. Further any such curve can only intersect the planes $x= \pm y$ at fixed points of type (F1) or type (F2) and these are bifurcation points. We define a type (F3) curve to be such a curve that does not intersect the planes $x= \pm y$ at all. Type (F4) curves are those that do intersect the $x= \pm y$ planes (at a bifurcation point). As indicated above we will actually show that type (F4) curves do not exist (by showing that $K_{n}(x, x) \neq 0$ for $x \in[-1,1])$. 


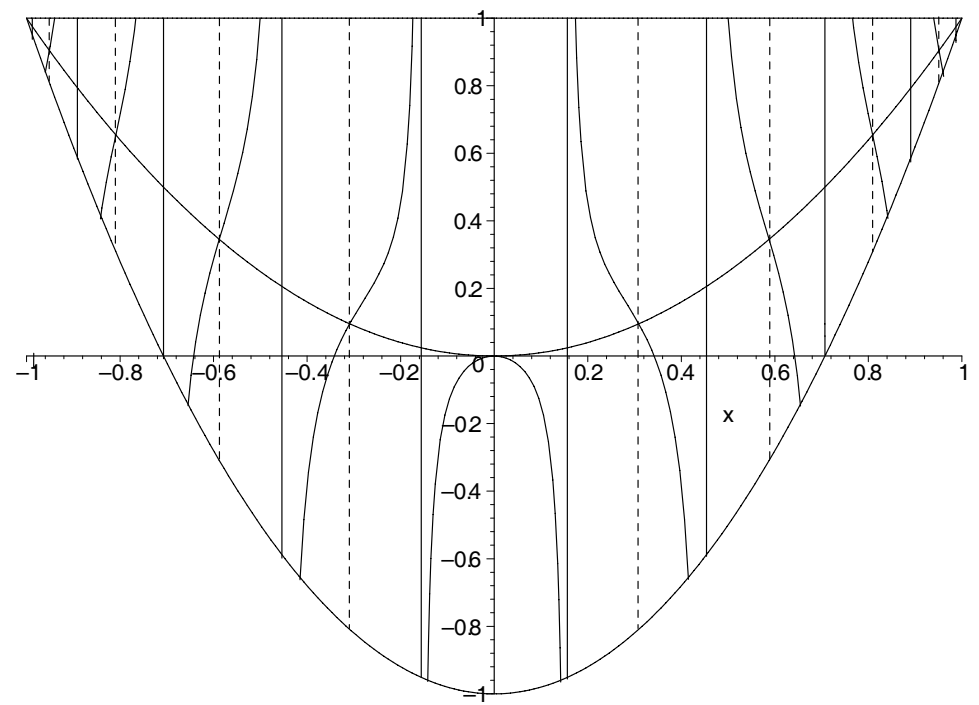

FIGURE 1 . The curves of fixed points of type (F1) and (F2) in the slice $x=y$.

In $\S 2$, we will define and examine the (F1) curves of fixed points. We find there that the Chebyshev polynomials $U_{n}(x)$ are needed for this investigation and we also give various properties of the $U_{n}$. We also prove (1.2) in $\S 1$. In $\S 3$, we define and study the fixed curves of type (F2) and show how the situation in the $x=y$ plane is similar to that in the $x=-y$ plane. In $\S 4$, we find the polynomial $K_{n}(x, y)$ and list some of its properties. We give a general explanation of $\alpha_{n}$-duality. We also determine the preserving and reversing fixed points and show that there are no curves of fixed points of type (F4). In $\$ 5$, we consider the more general case where $\alpha=\sigma_{1}^{r} \sigma_{2}^{s}, r, s \in \mathbb{N}$. In $\S 6$, we describe the $\alpha_{n}$-dual pairs of fixed points.

\section{The straight line curves: the (F1) case}

We first give a relatively simple form for the action of $\alpha_{n}$. This will involve Chebyshev polynomials. Define the Chebyshev polynomials $U_{n}(x)$ (see [Ri]) by

$$
U_{-1}(x)=0, \quad U_{0}(x)=1, \quad U_{1}(x)=2 x, \quad U_{n}(x)=2 x U_{n-1}(x)-U_{n-2}(x) .
$$

We have the following properties of $U_{n}(x)$ (see [Ri]):

$$
U_{n}(x)=2^{n} \prod_{k=1}^{n}\left(x-\cos \frac{k \pi}{n+1}\right), \quad U_{n}(\cos (\theta))=\frac{\sin ((n+1) \theta)}{\sin (\theta)} .
$$


LEMMA 2.1. If $n \in \mathbb{Z}$, then

$$
\begin{aligned}
& \left(\begin{array}{l}
x \\
y \\
z
\end{array}\right) \sigma_{1}^{k}=\left(\begin{array}{ccc}
0 & 0 & 1 \\
0 & 1 & 0 \\
-1 & 0 & 2 y
\end{array}\right)^{k}\left(\begin{array}{l}
x \\
y \\
z
\end{array}\right), \\
& \left(\begin{array}{l}
x \\
y \\
z
\end{array}\right) \sigma_{2}^{k}=\left(\begin{array}{ccc}
1 & 0 & 0 \\
0 & 2 x & -1 \\
0 & 1 & 0
\end{array}\right)^{k}\left(\begin{array}{l}
x \\
y \\
z
\end{array}\right) .
\end{aligned}
$$

Denote the above two $3 \times 3$ matrices by $M_{1}=M_{1}(y)$ and $M_{2}=M_{2}(x)$. Then we have

$$
\begin{aligned}
& M_{1}^{k}=\left(\begin{array}{ccc}
-U_{k-2}(y) & 0 & U_{k-1}(y) \\
0 & 1 & 0 \\
-U_{k-1}(y) & 0 & U_{k}(y)
\end{array}\right), \\
& M_{2}^{k}=\left(\begin{array}{ccc}
1 & 0 & 0 \\
0 & U_{k}(x) & -U_{k-1}(x) \\
0 & U_{k-1}(x) & -U_{k-2}(x)
\end{array}\right) .
\end{aligned}
$$

Proof. The first statement follows from the action of $\sigma_{i}, i=1,2$, given in (1.1) and the second follows by induction on $k \geq 1$ using the recursion for $U_{n}$ given in (2.1).

Corollary 2.2. For all $n \in \mathbb{N}$ and $(x, y, z)^{\mathrm{T}} \in \mathbb{R}^{3}$ we have

$$
\left(\begin{array}{l}
x \\
y \\
z
\end{array}\right) \alpha_{n}=\left(\begin{array}{c}
-x U_{n-2}(y)+z U_{n-1}(y) \\
U_{n}\left(x^{*}\right) y-U_{n-1}\left(x^{*}\right)\left[-x U_{n-1}(y)+z U_{n}(y)\right] \\
U_{n-1}\left(x^{*}\right) y-U_{n-2}\left(x^{*}\right)\left[-x U_{n-1}(y)+z U_{n}(y)\right]
\end{array}\right) .
$$

Here $x^{*}=-x U_{n-2}(y)+z U_{n-1}(y)$.

In particular, if $(x, y, z)^{\mathrm{T}} \in \mathbb{R}^{3}$ is a fixed point of $\alpha_{n}$ and $U_{n-1}(y) \neq 0$, then we must have

$$
z=x\left(1+U_{n-2}(y)\right) / U_{n-1}(y) .
$$

Proof. To prove the first statement we put $k=n$ in Lemma 2.1 and then multiply the matrices $M_{2}^{n}\left(x^{*}\right) M_{1}^{n}(y)(x, y, z)^{\mathrm{T}}$.

The last statement follows from the first by solving $-x U_{n-2}(y)+z U_{n-1}(y)=x$ for $z$.

We now consider the straight line cases. Let $I_{n}$ represent the $n \times n$ identity matrix.

LEMMA 2.3. Let $n, j, k \in \mathbb{N}, j, k \neq \equiv 0 \bmod n / 2$, and $a=\cos (2 \pi j / n)$.

(i) We have $M_{1}^{n}(a)=M_{2}^{n}(a)=I_{3}$.

(ii) If $\alpha \in\left\langle\sigma_{1}^{n}, \sigma_{2}^{n}\right\rangle$ and $v=(\cos (2 \pi j / n), \cos (2 \pi k / n), z)^{\mathrm{T}}$, then $(v) \alpha=v$. In particular, we have $(v) \alpha_{n}=v$ and the vertical line $\gamma(z)=(\cos (2 \pi j / n), \cos (2 \pi k / n), z)^{\mathrm{T}}$ is a curve of fixed points of $\alpha_{n}$.

(iii) If $j \equiv 0 \bmod n / 2$ or $k \equiv 0 \bmod n / 2$, then $(\cos (2 \pi j / n), \cos (2 \pi k / n), z)^{\mathrm{T}}$ is not $a$ curve of fixed points of $\alpha_{n}$.

Proof. From Lemma 2.1 we have

$$
M_{1}^{n}(a)=\left(\begin{array}{ccc}
-U_{n-2}(a) & 0 & U_{n-1}(a) \\
0 & 1 & 0 \\
-U_{n-1}(a) & 0 & U_{n}(a)
\end{array}\right),
$$


and using (2.2) we have (since $j \not \equiv 0 \bmod n / 2$ implies that $\sin (2 \pi j / n) \neq 0$ )

$$
\begin{aligned}
U_{n}(\cos (2 \pi j / n)) & =\sin ((n+1) 2 \pi j / n) / \sin (2 \pi j / n)=1, \\
U_{n-1}(\cos (2 \pi j / n)) & =\sin (n 2 \pi j / n) / \sin (2 \pi j / n)=0, \\
U_{n-2}(\cos (2 \pi j / n)) & =\sin ((n-1) 2 \pi j / n) / \sin (2 \pi j / n)=-1,
\end{aligned}
$$

which gives $M_{1}^{n}(a)=I_{3}$. The proof that $M_{2}^{n}(a)=I_{3}$ is similar.

Now from Lemma 2.1 and the above, we have $v \sigma_{1}^{n}=M_{1}^{n}(\cos (2 \pi k / n)) v=I_{3} v=v$ and $v \sigma_{2}^{n}=M_{2}^{n}(\cos (2 \pi j / n)) v=v$. It follows that

$$
v \alpha_{n}=\left(v \sigma_{1}^{n}\right) \sigma_{2}^{n}=v \sigma_{2}^{n}=v .
$$

This does (i) and (ii).

For (iii) we first do the case $k=n / 2$. Here we have $v=(\cos (2 \pi j / n),-1, z)^{\mathrm{T}}$ and from Corollary 2.2 we see that the first coordinate of $v \alpha_{n}$ is

$$
-\cos (2 \pi j / n) U_{n-2}(-1)+U_{n-1}(-1) z=-(n-1) \cos (2 \pi j / n)-n z \neq \cos (2 \pi j / n) .
$$

Here we have used the fact that for all $r \geq 1$ we have $U_{r}(-1)=(-1)^{r}(r+1)$, which is easily proved by induction (see also Proposition 2.6(v)). If $k=0$, then $v=(\cos (2 \pi j / n), 1, z)^{\mathrm{T}}$ and we similarly see that the first coordinate of $v \alpha_{n}$ is $n z-(n-1) \cos (2 \pi j / n) \neq$ $\cos (2 \pi j / n)$. The cases where $j \equiv 0, n / 2 \bmod n$ are similar.

LEMma 2.4. Any $\beta \in B_{3}$ which is in the normal closure of the subgroup $\left\langle\sigma_{1}^{4}, \sigma_{2}^{4}\right\rangle$ (which has finite index in $B_{3}$ ) fixes the axes $X, Y, Z$ pointwise.

In particular, if $n \in \mathbb{N}$ is a multiple of 4 , then $\alpha_{n}$ fixes these axes pointwise.

Proof. The fact that this normal closure has finite index is a consequence of applying the Reidemeister-Schreier algorithm [BC].

Since $\sigma_{1}$ and $\sigma_{2}$ are conjugate in $B_{3}$ it will suffice to show that the action of $\sigma_{1}^{4}$ fixes each point of $X, Y, Z$. If $(a, 0,0)^{\mathrm{T}} \in X \subset \mathbb{R}^{3}$, then

$$
(a, 0,0)^{\mathrm{T}} \sigma_{1}^{4}=(0,0,-a)^{\mathrm{T}} \sigma_{1}^{3}=(-a, 0,0)^{\mathrm{T}} \sigma_{1}^{2}=(0,0, a)^{\mathrm{T}} \sigma_{1}=(a, 0,0)^{\mathrm{T}},
$$

as required; this does the $x$-axis case and the rest are similar. The first result follows and the second is a direct consequence since $\alpha_{n}=\sigma_{1}^{n} \sigma_{2}^{n}$.

This concludes our discussion of the straight line curves of fixed points for $\alpha_{n}$.

The similarity between what happens on $\mathcal{T}_{+}$and $\mathcal{T}_{-}$, where $\mathcal{T}_{-}$is the intersection of $\mathcal{T}$ and the plane $x=-y$, is indicated in the following result.

\section{PROPOSITION 2.5.}

(i) The involutive automorphism

$$
S: \mathbb{Q}[x, y, z] \rightarrow \mathbb{Q}[x, y, z], \quad x \mapsto-x, y \mapsto y, z \mapsto-z,
$$

centralizes any $\alpha \in\left\langle\sigma_{1}, \sigma_{2}^{2}\right\rangle$, that is $\alpha S=S \alpha$. In particular, if $n$ is even, then $S$ centralizes $\alpha_{n}$.

(ii) The involutive automorphism

$$
R: \mathbb{Q}[x, y, z] \rightarrow \mathbb{Q}[x, y, z], \quad x \mapsto y, y \mapsto x, z \mapsto z,
$$

conjugates $\sigma_{2}$ to $\sigma_{1}^{-1}$. The map $R$ reverse centralizes $\alpha_{n}$ so that $\alpha_{n} R=R \alpha_{n}^{-1}$. 
Proof. (i) We have

$$
\begin{aligned}
& \sigma_{1} S(x, y, z)=\sigma_{1}(-x, y,-z)=(-z, y,-(2 y z-x)), \\
& S \sigma_{1}(x, y, z)=S(z, y, 2 y z-x)=(-z, y,-2 y z+x),
\end{aligned}
$$

showing that $S$ centralizes $\sigma_{1}$. The calculation showing that $S$ centralizes $\sigma_{2}^{2}$ is similar. Thus $S$ centralizes any element of $H=\left\langle\sigma_{1}, \sigma_{2}^{2}\right\rangle$, and if $n$ is even, then $\alpha_{n} \in H$.

For (ii) we have

$$
\begin{aligned}
R \sigma_{2} R \sigma_{1}(x, y, z) & =R \sigma_{2} R(z, y, 2 y z-x)=R \sigma_{2}(z, x, 2 x z-y) \\
& =R(y, x, 2 x y-(2 x y-z))=(x, y, z),
\end{aligned}
$$

as required. It follows that

$$
R \sigma_{1}^{n} \sigma_{2}^{n} R=\sigma_{2}^{-n} \sigma_{1}^{-n}=\left(\sigma_{1}^{n} \sigma_{2}^{n}\right)^{-1} .
$$

Throughout this paper we will have need of the following properties of Chebyshev polynomials. For all $n \geq 0$ and $a \in \mathbb{R}$ we will let $U_{n}^{\prime}(a)$ denote

$$
\left.\frac{d}{d x} U_{n}(x)\right|_{x=a} \text {. }
$$

Proposition 2.6. For $m \in \mathbb{N}$ we have the following:

(i) $U_{m}(-x)=(-1)^{m} U_{m}(x)$;

(ii) $U_{m}(1)=m+1$;

(iii) $U_{2 m}^{\prime}(0)=0$;

(iv) $U_{2 m+1}^{\prime}(0)=(-1)^{m} 2(m+1)$;

(v) $U_{m}(-1)=(-1)^{m}(m+1)$;

(vi) $U_{m-1}^{2}-U_{m} U_{m-2}=1$;

(vii) $U_{m}(x)^{2}-2 x U_{m-1}(x) U_{m}(x)+U_{m-1}(x)^{2}=1$;

(viii) $U_{2 m}(x)=U_{m}(x)^{2}-U_{m-1}(x)^{2}$;

(ix) $U_{2 m-1}(x)=2 U_{m} U_{m-1}-2 x U_{m-1}^{2}$;

(x) for all even $n>1$ we have $\operatorname{gcd}\left(1+U_{n-2}(x), U_{n-1}(x)\right)=U_{n / 2-1}(x)$;

(xi) $U_{m}^{\prime}(1)=2\left(\begin{array}{c}m+2 \\ 3\end{array}\right)$.

Proof. (i)-(v) These follow easily from the definitions, sometimes by induction.

(vi) This follows from the fact that $\operatorname{det} M_{1}=1$ and so det $M_{1}^{m}=U_{m-1}^{2}-U_{m} U_{m-2}=1$ (see the representations of $M_{1}$ and $M_{1}^{m}$ in Lemma 2.1).

(vii) This follows from (vi) after substituting $2 x U_{m-1}(x)=U_{m}(x)+U_{m-2}(x)$ into (vi).

(viii) and (ix) The proof of these is by simultaneous induction, the initial cases being easy to check. If (viii) and (ix) are true, then (using (2.1)) we have

$$
\begin{aligned}
U_{2 m+1} & =2 x U_{2 m}-U_{2 m-1} \\
& =2 x\left(U_{m}^{2}-U_{m-1}^{2}\right)-\left(2 U_{m} U_{m-1}-2 x U_{m-1}^{2}\right) \\
& =2 x U_{m}^{2}-2 U_{m} U_{m-1} \\
& =2 x U_{m}^{2}-2 U_{m}\left(2 x U_{m}-U_{m+1}\right) \\
& =2 U_{m+1} U_{m}-2 x U_{m}^{2},
\end{aligned}
$$


as required for (ix). Also,

$$
\begin{aligned}
U_{2(m+1)} & =2 x U_{2 m+1}-U_{2 m} \\
& =2 x\left(2 U_{m+1} U_{m}-2 x U_{m}^{2}\right)-U_{m}^{2}+U_{m-1}^{2} \\
& =2 x\left(2 U_{m+1} U_{m}-2 x U_{m}^{2}\right)-U_{m}^{2}+\left(2 x U_{m}-U_{m+1}\right)^{2} \\
& =U_{m+1}^{2}-U_{m}^{2} .
\end{aligned}
$$

This does (viii) and (ix).

(x) For this we first note that every root of $U_{n / 2-1}(x)$ is a root of $U_{n-1}(x)$ (see (ix) above). Next, any root of $U_{n / 2-1}$ has the form $\cos (2 \pi k /(n / 2))$ and so by (2.2) we get

$$
U_{n-2}(\cos (2 \pi k /(n / 2)))=\sin ((n-1) 2 \pi k /(n / 2)) / \sin (2 \pi k /(n / 2))=-1 \text {, }
$$

which shows that $\cos (2 \pi k /(n / 2))$ is a root of $1+U_{n-2}(x)$. The fact that these are the only values of $x=\cos (2 \pi k / n)$ (the roots of $\left.U_{n-1}(x)\right)$ that give $1+U_{n-2}(x)=0$ gives the rest of (x).

(xi) This is proved by induction on $m \geq 1$ using the recursion (2.1).

We will need to define the T-type Chebyshev polynomials:

$$
T_{0}=1, \quad T_{1}(x)=x, \quad T_{n}(x)=2 x T_{n-1}(x)-T_{n-2}(x) .
$$

LEMMA 2.7. For $n \geq 0$ we have

$$
U_{n}^{\prime}=\sum_{k=0, k \equiv n(2)}^{n} 2 k U_{k-1} .
$$

Proof. First assume that $n=2 k$ is even. Then from [Ri, p. 7, equation (1.23)] we have $T_{n}^{\prime}(x)=n U_{n-1}(x)$ and from [Ri, p. 8, Exercise 1.2.13] we have $U_{2 k}=1+2 T_{2}+2 T_{4}+$ $2 T_{6}+\cdots+2 T_{2 k}$ so that

$$
U_{2 k}^{\prime}=2\left(T_{2}^{\prime}+T_{4}^{\prime}+T_{6}^{\prime}+\cdots+T_{2 k}^{\prime}\right)=2\left(2 U_{1}+4 U_{3}+6 U_{5}+\cdots+2 k U_{2 k-1}\right),
$$

as required for the $n$ even case. The $n=2 k+1$ case is similar, only one uses [Ri, p. 9, Exercise 1.2.15]: $U_{2 k+1}=2\left(T_{1}+T_{3}+T_{5}+\cdots+T_{2 k+1}\right)$.

For $\alpha \in B_{3}, p \in \mathbb{R}^{3}$, we let $J_{p}(\alpha)$ denote the Jacobian of $\alpha$ at $p$.

\section{LEMMA 2.8.}

(i) For $x \in \mathbb{R}$ the minimum value of $E$ along the curve $p(z)=(x, x, z)^{\mathrm{T}}$ is attained where $z=x^{2}$.

(ii) For $x, y \in \mathbb{R}$ the minimum value of $E$ along the curve $p(z)=(x, y, z)^{\mathrm{T}}$ is attained where $z=x y$.

Proof. Clearly (i) follows from (ii). For (ii) we differentiate $E(x, y, z)$ with respect to $z$, which gives $2 z-2 x y$ and the result follows.

We next justify the formula (1.2). 
LEMMA 2.9. For all $\alpha \in B_{3}$ there is a matrix $\Phi(\alpha) \in S L(2, \mathbb{Z})$ such that

$$
\left(\Pi\left(\left(\begin{array}{l}
\theta_{1} \\
\theta_{2}
\end{array}\right)\right)\right) \alpha=\Pi\left(\Phi(\alpha)\left(\begin{array}{l}
\theta_{1} \\
\theta_{2}
\end{array}\right)\right) .
$$

In particular

$$
\Phi\left(\sigma_{1}\right)=\left(\begin{array}{ll}
1 & 1 \\
0 & 1
\end{array}\right), \quad \Phi\left(\sigma_{2}\right)=\left(\begin{array}{rr}
1 & 0 \\
-1 & 1
\end{array}\right) .
$$

For $\alpha, \beta \in B_{3}$ we have

$$
\left(\Pi\left(\begin{array}{l}
\theta_{1} \\
\theta_{2}
\end{array}\right)\right) \alpha \beta=\Pi\left(\Phi(\beta) \Phi(\alpha)\left(\begin{array}{l}
\theta_{1} \\
\theta_{2}
\end{array}\right)\right) .
$$

Lastly, we have

$$
\Phi\left(\alpha_{n}\right)=\left(\begin{array}{cc}
1 & n \\
-n & 1-n^{2}
\end{array}\right)
$$

Proof. For $\alpha=\sigma_{1}^{ \pm 1}, \sigma_{2}^{ \pm 1}$ this is a calculation. For example consider $\sigma_{1}$ : if $(x, y, z)^{\mathrm{T}}=$ $\Pi\left(\theta_{1}, \theta_{2}\right)^{\mathrm{T}}$, so that $x=\cos \left(2 \pi \theta_{1}\right)$ etc., then we let

$$
m_{1}=\left(\begin{array}{cc}
e^{2 \pi i \theta_{1}} & 0 \\
0 & e^{-2 \pi i \theta_{1}}
\end{array}\right), \quad m_{2}=\left(\begin{array}{cc}
e^{2 \pi i \theta_{2}} & 0 \\
0 & e^{-2 \pi i \theta_{2}}
\end{array}\right)
$$

so that

$$
x=\operatorname{trace}\left(m_{1}\right) / 2, \quad y=\operatorname{trace}\left(m_{2}\right) / 2, \quad z=\operatorname{trace}\left(m_{1} m_{2}\right) / 2 .
$$

Now $(x, y, z)^{\mathrm{T}} \sigma_{1}=(z, y, 2 y z-x)^{\mathrm{T}}$. But if we let

$$
\begin{gathered}
m_{1}^{\prime}=m_{1} m_{2}=\left(\begin{array}{cc}
e^{2 \pi i\left(\theta_{1}+\theta_{2}\right)} & 0 \\
0 & e^{-2 \pi i\left(\theta+\theta_{2}\right)}
\end{array}\right), \quad m_{2}^{\prime}=m_{2}=\left(\begin{array}{cc}
e^{2 \pi i \theta_{2}} & 0 \\
0 & e^{-2 \pi i \theta_{2}}
\end{array}\right), \\
m_{3}^{\prime}=\left(\begin{array}{cc}
e^{2 \pi i\left(\theta_{1}+2 \theta_{2}\right)} & 0 \\
0 & e^{-2 \pi i\left(\theta_{1}+2 \theta_{2}\right)}
\end{array}\right),
\end{gathered}
$$

then we have

$$
\operatorname{trace}\left(m_{1}^{\prime}\right) / 2=z, \quad \operatorname{trace}\left(m_{2}^{\prime}\right) / 2=y, \quad \operatorname{trace}\left(m_{3}^{\prime}\right) / 2=2 y z-x,
$$

showing that the effect of $\sigma_{1}$ on $\left(\theta_{1}, \theta_{2}\right)^{\mathrm{T}}$ is that of the matrix $\left(\begin{array}{ll}1 & 1 \\ 0 & 1\end{array}\right)$. The proof for $\sigma_{2}$ is similar.

The result for general $\alpha$ follows by induction on the length of $\alpha$ as a word in these generators.

Let $\alpha, \beta \in B_{3}$; then from the above we have

$$
\left(\Pi\left(\begin{array}{l}
\theta_{1} \\
\theta_{2}
\end{array}\right)\right) \alpha \beta=\left(\Pi \Phi(\alpha)\left(\begin{array}{l}
\theta_{1} \\
\theta_{2}
\end{array}\right)\right) \beta=\Pi\left(\Phi(\beta) \Phi(\alpha)\left(\begin{array}{l}
\theta_{1} \\
\theta_{2}
\end{array}\right)\right)
$$

as required.

Lastly

$$
\Phi\left(\alpha_{n}\right)=\Phi\left(\sigma_{1}^{n} \sigma_{2}^{n}\right)=\Phi\left(\sigma_{2}^{n}\right) \Phi\left(\sigma_{1}^{n}\right)=\left(\begin{array}{cc}
1 & 0 \\
-n & 1
\end{array}\right)\left(\begin{array}{cc}
1 & n \\
0 & 1
\end{array}\right)=\left(\begin{array}{cc}
1 & n \\
-n & 1-n^{2}
\end{array}\right),
$$

as required.

We next indicate how fixed points $p$ of $\alpha_{n}$ on $\partial \mathcal{T} \backslash V$ give rise to a curve of fixed points containing $p$. We use this result throughout this paper. 
LEMMA 2.10. [Ro p. 160, PM p. 55] Let $\beta \in B_{3}$ where $\Phi(\beta)$ is hyperbolic. If $p \in \partial \mathcal{T} \backslash V \subset E_{1}$ is a fixed point for $\beta$, then there is a $C^{\infty}$ curve $\gamma:(-1,1) \rightarrow \mathbb{R}^{3}$ such that:

(i) $\quad \gamma(t)$ is fixed by $\beta$ for every $t \in(-1,1)$;

(ii) $\gamma(0)=p$;

(iii) there is $\varepsilon>0$ such that for all $t \in(-\varepsilon, \varepsilon)$ we have $\gamma(t) \in E_{1+t}$.

After having for the most part ignored the points in $V$ we now describe what happens near those points.

LEMMA 2.11. Let $n$ be even and put $p=(1,1,1)^{\mathrm{T}}$. Then the points of $V$ are fixed by the normal closure of $\left\langle\sigma_{1}^{2}, \sigma_{2}^{2}\right\rangle$. We have

$$
J_{p}\left(\sigma_{1}^{n}\right)=\left(\begin{array}{ccc}
1-n & n(n-1) & n \\
0 & 1 & 0 \\
-n & n(n+1) & n+1
\end{array}\right), \quad J_{p}\left(\sigma_{2}^{n}\right)=\left(\begin{array}{ccc}
1 & 0 & 0 \\
n(n+1) & n+1 & -n \\
n(n-1) & n & 1-n
\end{array}\right) .
$$

We also have trace $\left(J_{p}\left(\alpha_{n}\right)\right)=3+n^{2}\left(n^{2}-4\right)$ and $J_{p}\left(\alpha_{n}\right)$ has $(1,1,2-n)^{\mathrm{T}}$ as an eigenvector with eigenvalue 1.

Further, the points $(1,1,1)^{\mathrm{T}}$ and $(-1,-1,1)^{\mathrm{T}}$ are on a part of the curve $\gamma_{+}(x)=$ $\left(x, x, x\left(1+U_{n-2}(x)\right) / U_{n-1}(x)\right)^{\mathrm{T}}$ of fixed points which does not intersect the interior of the disc $\mathcal{T}_{+}$.

The behaviour at the other points $q \in V$ is similar, in particular we have $\operatorname{trace}\left(J_{q}\left(\alpha_{n}\right)\right)=3+n^{2}\left(n^{2}-4\right)$.

Proof. It is easy to check that $q \sigma_{k}^{2}=q$ for $k=1,2$ and $q \in V$. Since $n$ is even the point $p$ is fixed by $\sigma_{1}^{n}$ and by $\sigma_{2}^{n}$. From Lemma 2.1 we obtain

$$
(x, y, z)^{\mathrm{T}} \sigma_{1}^{n}=\left(-x U_{n-2}(y)+z U_{n-1}(y), y,-x U_{n-1}(y)+z U_{n}(y)\right)^{\mathrm{T}},
$$

so that using Proposition 2.6 we have

$$
\begin{aligned}
J_{p}\left(\sigma_{1}^{n}\right) & =\left(\begin{array}{ccc}
-U_{n-2} & -x U_{n-2}^{\prime}+z U_{n-1}^{\prime} & U_{n-1} \\
0 & 1 & 0 \\
-U_{n-1} & -x U_{n-1}^{\prime}+z U_{n}^{\prime} & U_{n}
\end{array}\right) \\
& =\left(\begin{array}{ccc}
1-n & -2\left(\begin{array}{c}
n \\
3
\end{array}\right)+2\left(\begin{array}{c}
n+1 \\
3
\end{array}\right) & n \\
0 & 1 & 0 \\
-n & -2\left(\begin{array}{c}
n+1 \\
3
\end{array}\right)+2\left(\begin{array}{c}
n+2 \\
3
\end{array}\right) & n+1
\end{array}\right),
\end{aligned}
$$

which one can check is what we want. The calculation of $J_{p}\left(\sigma_{2}^{n}\right)$ is similar.

Now since $\sigma_{1}^{n}$ and $\sigma_{2}^{n}$ both fix $p$ we have

$$
\begin{aligned}
J_{p}\left(\alpha_{n}\right) & =J_{p}\left(\sigma_{2}^{n}\right) J_{p}\left(\sigma_{1}^{n}\right) \\
& =\left(\begin{array}{ccc}
1-n & n(n-1) & n \\
n-n^{3}+n^{2} & 1+n-2 n^{2}-n^{3}+n^{4} & n\left(n^{2}-1\right) \\
3 n^{2}-n^{3}-2 n & n^{4}-3 n^{3}+n^{2}+2 n & 1+n^{2}(n-2)
\end{array}\right) .
\end{aligned}
$$

The trace is now easily calculated, as is the fact that $(1,1,2-n)^{\mathrm{T}}$ is an eigenvector for eigenvalue 1 . 
Lastly, when $x=1$ we see from Proposition 2.6 that

$$
x\left(1+U_{n-2}(x)\right) / U_{n-1}(x)=(1+(n-1)) / n=1,
$$

which shows that $(1,1,1)^{\mathrm{T}}$ is on the curve $\left(x, x, x\left(1+U_{n-2}(x)\right) / U_{n-1}(x)\right)^{\mathrm{T}}$. To see that this curve does not intersect the interior of $\mathcal{T}_{+}$near $(1,1,1)^{\mathrm{T}}$ we just note that the tangent vector at $(1,1,1)^{\mathrm{T}}$ to such a curve is the above-mentioned eigenvector $(1,1,2-n)^{\mathrm{T}}$ and in the plane $x=y$ this has negative slope, since $n>2$. Thus it does not enter $\mathcal{T}_{+}$near $(1,1,1)^{\mathrm{T}}$. See Figure 1 .

The proof for $(-1,-1,1)^{\mathrm{T}}$ is similar.

Now $\Pi\left(\theta_{1}, \theta_{2}\right)^{\mathrm{T}}=(x, x, z)^{\mathrm{T}}$ if and only if $\theta_{1}= \pm \theta_{2}$.

LEMMA 2.12. The $\alpha_{n}$-fixed points

$$
\begin{aligned}
\Pi(i / n, j / n)^{\mathrm{T}} & =(\cos (2 \pi i / n), \cos (2 \pi j / n), \cos (2 \pi(i+j) / n))^{\mathrm{T}}, \\
\Pi(i / n,-j / n)^{\mathrm{T}} & =(\cos (2 \pi i / n), \cos (-2 \pi j / n), \cos (2 \pi(i-j) / n))^{\mathrm{T}},
\end{aligned}
$$

on $\partial \mathcal{T}$, are either equal or are joined by a vertical line of fixed points in the interior of $\mathcal{T}$ and are $\alpha_{n}$-dual points.

Proof. By Lemma $2.3(x, y, z)^{\mathrm{T}} \alpha_{n}=(x, y, z)^{\mathrm{T}}$ if $x=\cos (2 \pi i / n), y=\cos ( \pm 2 \pi j / n)$. With these values of $x, y$ the values $z_{ \pm}=\cos (2 \pi(i \pm j) / n)$ are the two solutions (for $z$ ) of the equation $x^{2}+y^{2}+z^{2}-2 x y z=1$ and so we get the two indicated points on the boundary of $\mathcal{T}$. The result follows.

3. The $x= \pm y$ curves of fixed points: the (F2) case

In this section we first show that the curve $\gamma_{+}(x)$ (see (1.3)) is a curve of fixed points for $\alpha_{n}$. We will need the following fact about Chebyshev polynomials.

LEMMA 3.1. For all $n \geq 2$ we have

$$
U_{n}^{2}-2 x U_{n} U_{n-1}+U_{n-1}^{2}=1 .
$$

Proof. This is by induction on $n \geq 2$ where we check the first case and then note that, using the recurrence (2.1), we get

$$
\begin{aligned}
U_{n}^{2} & -2 x U_{n} U_{n-1}+U_{n-1}^{2} \\
& =\left(2 x U_{n-1}-U_{n-2}\right)^{2}-2 x\left(2 x U_{n-1}-U_{n-2}\right) U_{n-1}+U_{n-1}^{2} \\
& =U_{n-1}^{2}-2 x U_{n-1} U_{n-2}+U_{n-2}^{2}=1,
\end{aligned}
$$

as required.

LEMMA 3.2 .

(i) For all $n \geq 1$ we have

$$
\left(x, x, x\left(1+U_{n-2}(x)\right) / U_{n-1}(x)\right)^{\mathrm{T}} \sigma_{1}^{n}=\left(x, x, 2 x^{2}-x\left(1+U_{n-2}(x)\right) / U_{n-1}(x)\right)^{\mathrm{T}} .
$$

Further, we also have

$$
\left(x, x, 2 x^{2}-x\left(1+U_{n-2}(x)\right) / U_{n-1}(x)\right)^{\mathrm{T}} \sigma_{2}^{n}=\left(x, x, x\left(1+U_{n-2}(x)\right) / U_{n-1}(x)\right)^{\mathrm{T}} .
$$


For all $n \in \mathbb{N}$ the curve $\gamma_{+}(x)=\left(x, x, x\left(1+U_{n-2}(x)\right) / U_{n-1}(x)\right)^{\mathrm{T}}$ is fixed by $\alpha_{n}=\sigma_{1}^{n} \sigma_{2}^{n}$.

(ii) For all $n \in 2 \mathbb{N}$ the curve $\gamma_{-}(x)=\left(-x, x,-x\left(1+U_{n-2}(x)\right) / U_{n-1}(x)\right)^{\mathrm{T}}$ is fixed by $\alpha_{n}$.

Proof. (i) The last statement follows from the first two, which we now prove. By Lemma 2.1 we have

$$
\begin{aligned}
& \left(\begin{array}{c}
x \\
x \\
x \\
x\left(1+U_{n-2}(x)\right) / U_{n-1}(x)
\end{array}\right) \sigma_{1}^{n} \\
& \quad=\left(\begin{array}{ccc}
-U_{n-2}(x) & 0 & U_{n-1}(x) \\
0 & 1 & 0 \\
-U_{n-1}(x) & 0 & U_{n}(x)
\end{array}\right)\left(\begin{array}{c}
x \\
x \\
x\left(1+U_{n-2}(x)\right) / U_{n-1}(x)
\end{array}\right) \\
& =\left(\begin{array}{c}
x \\
-x U_{n-2}(x)+x\left(1+U_{n-2}(x)\right) \\
-x U_{n-1}(x)+x U_{n}(x)\left(1+U_{n-2}(x)\right) / U_{n-1}(x)
\end{array}\right) .
\end{aligned}
$$

Substituting for $U_{n}(x)$ using (2.1) this latter vector is

$$
\left(\begin{array}{c}
x \\
x \\
2 x^{2}+\left[-x U_{n-1}^{2}(x)+2 x^{2} U_{n-1}(x) U_{n-2}(x)-x U_{n-2}-x U_{n-2}^{2}\right] / U_{n-1}(x)
\end{array}\right) .
$$

For the first part of Lemma 3.2(i) it thus suffices to show that

$$
-x U_{n-1}^{2}(x)+2 x^{2} U_{n-1}(x) U_{n-2}(x)-x U_{n-2}-x U_{n-2}^{2}=-x\left(1+U_{n-2}(x)\right),
$$

and after simplification this reduces to showing that

$$
1=U_{n-1}^{2}(x)-2 x U_{n-1}(x) U_{n-2}(x)+U_{n-2}^{2}(x),
$$

which itself follows from Lemma 3.1.

This proves the first result in Lemma 3.2(i) and the second is similar. The last statement of (i) follows directly.

(ii) The proof here is similar to that of (i). Let $H_{n}(x)=x\left(1+U_{n-2}(x)\right) / U_{n-1}(x)$. We first show that $\left(-x, x,-H_{n}(x)\right)^{\mathrm{T}} \sigma_{1}^{n}=\left(-x, x,-2 x^{2}-H_{n}(x)\right)^{\mathrm{T}}$ and then that $\left(-x, x,-2 x^{2}+H_{n}(x)\right)^{\mathrm{T}} \sigma_{2}^{n}=\left(-x, x,-H_{n}(x)\right)^{\mathrm{T}}$. This gives (ii).

By Proposition 2.5 we see that if $(a, a, c)^{\mathrm{T}} \in \mathcal{T}_{+}$is a fixed point of $\alpha_{n}$, then $(-a, a,-c)^{\mathrm{T}} \in \mathcal{T}_{-}$is a fixed point of $\alpha_{n}$ and vice versa. This is why we need only consider $\mathcal{T}_{+}$. In Lemma 3.6 we will show that the above result determines all fixed points in $\mathcal{T}_{+}$.

LEMMA 3.3. The action of $B_{3}=\left\langle\sigma_{1}, \sigma_{2}\right\rangle$ on $\mathbb{R}^{3}$ is volume preserving.

Proof. Let $\alpha \in B_{3}$ and suppose that $\alpha$ has length $n$ as a word in $\sigma_{1}^{ \pm 1}, \sigma_{2}^{ \pm 1}$. We prove Lemma 3.3 by showing that $\operatorname{det} J_{p}(\alpha)=1$. We do this by induction on $n \geq 0$, the cases $n=0,1$ being easy to check using Lemma 2.1 and the general result following from the chain rule. 
We now investigate the roots and poles of the function $\left(1+U_{n-2}(x)\right) / U_{n-1}(x)$.

Define the polynomials

$$
Y_{n}(x)=x U_{n}-U_{n-1}-x .
$$

LEMMA 3.4. Let $n$ be even. Then the polynomial $Y_{n}$ has degree $n+1$. The roots of $Y_{n}(x)$ are

$$
\begin{aligned}
\cos \left(\frac{\pi k}{n}\right), & k=0,2,4, \ldots, n ; \\
\cos \left(\frac{\pi k}{n+2}\right), \quad k & =2,4,6, \ldots, n .
\end{aligned}
$$

Proof. Since $U_{n}(x)$ has degree $n$ the first statement follows. We thus need to show that the numbers in the above list are roots of $Y_{n}(x)$, since they are all distinct and there are $n+1$ of them.

First note that from Proposition 2.6 we have $U_{m}(1)=m+1$ and so when $k=0$ we have $\cos (\pi k / n)=1$ and $Y_{n}(1)=U_{n}(1)-U_{n-1}(1)-1=n+1-n-1=0$ as required. Thus we may assume that $k>0$.

Similarly when $k=n$ we have $x=\cos (\pi n / n)=-1$ and we again see using Proposition 2.6 that $Y_{n}(-1)=0$. Thus we assume that $k \neq n$. This then means that $\sin (\pi k / n) \neq 0$, which we use in what follows.

From (2.2) we see that $x=\cos \theta$ is a root of $Y_{n}$ if and only if

$$
Y_{n}(x)=\cos \theta \frac{\sin [(n+1) \theta]}{\sin \theta}-\cos \theta-\frac{\sin (n \theta)}{\sin \theta}
$$

is equal to 0 . Now for $k$ even, $x=\cos \theta$ and $\theta=\pi k / n$ we see that

$$
\begin{aligned}
Y_{n}(x) & =\cos (\pi k / n) \frac{\sin [(n+1) \pi k / n]}{\sin (\pi k / n)}-\cos (\pi k / n)-\frac{\sin (n \pi k / n)}{\sin (\pi k / n)} \\
& =\cos (\pi k / n) \frac{\sin (\pi k / n)}{\sin (\pi k / n)}-\cos (\pi k / n)-0 \\
& =0 .
\end{aligned}
$$

For the second case with $k$ even, $\theta=\pi k /(n+2)$ and $x=\cos \theta$ we have

$$
\begin{aligned}
Y_{n}(x) & =\cos \theta \frac{\sin [(n+1) \pi k /(n+2)]}{\sin \theta}-\cos \theta-\frac{\sin [n \pi k /(n+2)]}{\sin \theta} \\
& =\cos \theta \frac{\sin [-\pi k /(n+2)]}{\sin \theta}-\cos \theta-\frac{\sin [-2 \pi k /(n+2)]}{\sin \theta} \\
& =-\cos \theta \frac{\sin \theta}{\sin \theta}-\cos \theta-\frac{\sin (-2 \theta)}{\sin \theta}=0 .
\end{aligned}
$$

This proves Lemma 3.4.

We are now interested in finding those points of the tetrahedron $\partial \mathcal{T}$ that are also on the curve $\gamma_{+}(x)$ which is fixed by $\alpha_{n}=\sigma_{1}^{n} \sigma_{2}^{n}$. This will identify the $\alpha_{n}$-dual pairs of points on $\partial \mathcal{T}$ for $\gamma_{+}(x)$. 
Proposition 3.5. Let $n$ be even. Then the function $E\left(\gamma_{+}(x)\right)-1$ is equal to

$$
Y_{n}(x) Y_{n-2}(x) / U_{n-1}^{2} \text {. }
$$

The rational function $Y_{n}(x) Y_{n-2}(x) / U_{n-1}^{2}$ has roots

$$
\begin{aligned}
& \cos \frac{\pi k}{n+2}, \quad k=0,2,4, \ldots, n+2 ; \\
& \cos \frac{\pi k}{n-2}, \quad k=0,2,4, \ldots, n-2 .
\end{aligned}
$$

Except for \pm 1 all of these roots have multiplicity one.

It has poles of multiplicity two at $\cos (\pi k / n)$ for $k=1,3,5, \ldots, n-1$.

Let $n=2 m$ where $m$ is even. Then the following gives the ordered list of roots and poles (for any such fraction $r / s$ the corresponding root or pole is $\cos (\pi r / s)$ ):

$$
\begin{aligned}
1 & >\frac{n-1}{n}>\frac{n}{n+2}>\frac{n-4}{n-2}>\frac{n-3}{n}>\frac{n-2}{n+2}>\frac{n-6}{n-2}>\cdots \\
& >\frac{m+5}{n}>\frac{m+6}{n+2}>\frac{m+2}{n-2}>\frac{m+3}{n}>\frac{m+4}{n+2}>\frac{m}{n-2}>\frac{m+1}{n}>\frac{m+2}{n+2} \\
& >\frac{m}{n+2}>\frac{m-1}{n}>\frac{m-2}{n-2}>\frac{m-2}{n+2}>\cdots>\frac{6}{n+2}>\frac{5}{n}>\frac{4}{n-2}>\frac{4}{n+2} \\
& >\frac{3}{n}>\frac{2}{n-2}>\frac{2}{n+2}>\frac{1}{n}>0 .
\end{aligned}
$$

For $0 \leq k \leq m-4$ even, the points $\gamma_{+}(x), x=\cos [\pi(n-k) /(n+2)]$, $\cos [\pi(n-k-4) /(n-2)]$ are $\alpha_{n}$-dual points.

For $2 \leq k \leq m-2$ even, the points $\gamma_{+}(x), x=\cos [\pi k /(n-2)], \cos [\pi k /(n+2)]$ are $\alpha_{n}$-dual points.

Lastly, the points $\gamma_{+}(x), x=\cos [\pi m /(n+2)], \cos [\pi(m+2) /(n+2)]$ are $\alpha_{n}$-dual points.

Proof. The expression $E\left(\gamma_{+}(x)\right)-1$ is equal to

$$
\begin{aligned}
x^{2} & +x^{2}+x^{2}\left(1+U_{n-2}\right)^{2} / U_{n-1}^{2}-2 x^{3}\left(1+U_{n-2}\right) / U_{n-1}-1 \\
& =\left(2 x^{2} U_{n-1}^{2}+x^{2}\left(1+2 U_{n-2}+U_{n-2}^{2}\right)-2 x^{3}\left(1+U_{n-2}\right) U_{n-1}-U_{n-1}^{2}\right) / U_{n-1}^{2} \\
& =-\left(2 x^{2} U_{n-1}-x U_{n-2}-x-U_{n-1}\right)\left(x U_{n-2}+x-U_{n-1}\right) / U_{n-1}^{2} \\
& =-\left(x U_{n}-x-U_{n-1}\right)\left(x U_{n-2}+x-2 x U_{n-2}+U_{n-3}\right) / U_{n-1}^{2} \\
& =\left(x U_{n}-x-U_{n-1}\right)\left(x U_{n-2}-x-U_{n-3}\right) / U_{n-1}^{2} \\
& =Y_{n}(x) Y_{n-2}(x) / U_{n-1}^{2} .
\end{aligned}
$$

Now from Lemma 3.4 we see that $Y_{n}$ has roots

$$
\cos \frac{\pi k}{n}, \quad k=0,2,4, \ldots, n ; \quad \cos \frac{\pi k}{n+2}, \quad k=2,4, \ldots, n,
$$

while $Y_{n-2}$ has roots

$$
\cos \frac{\pi k}{n-2}, \quad k=0,2,4, \ldots, n-2 ; \quad \cos \frac{\pi k}{n}, \quad k=2,4, \ldots, n-2 .
$$


From (2.2) we see that $U_{n-1}^{2}$ has roots

$$
\cos \frac{\pi k}{n}, \quad k=1,2, \ldots, n-1,
$$

each with multiplicity two. The roots common to $Y_{n} Y_{n-2}$ and $U_{n-1}^{2}$ are thus $\cos (\pi k / n), k=2,4, \ldots, n-2$, each with multiplicity two. This part of the lemma now follows upon cancelling these roots.

The given ordering of the fractions is now easily checked.

Consider the first case: $0 \leq k \leq m-4$ even. From Lemma 2.8 we know that the vertical line in $\mathcal{T}_{+}$with $x=\cos ((n-k-2) \pi / n)$ bifurcates with $\gamma_{+}$at a single point. The vertical lines with $x=\cos ((n-k-1) \pi / n), x=\cos ((n-k-3) \pi / n)$ are poles for the function $\gamma_{+}$. From the above ordering there is a single point of $\partial \mathcal{T}_{+}$between these two poles and so they must be on the same component of the intersection of $\gamma_{+}$with $\mathcal{T}_{+}$. (It is useful to refer to Figure 1.)

The case $2 \leq k \leq m-2$ even is similar.

The centre of the above order list $(3.1)$ is $(m+2) /(n+2)>m /(n+2)$ which corresponds to the component of $\gamma_{+}(x)$ which has both of its end points near $z=-1$; see Figure 1 .

The above result indicates the places where the curve $\gamma_{+}(x)$ meets $\mathcal{T}_{+}$and also indicates the poles of $x\left(1+U_{n-2}(x)\right) / U_{n-1}(x)$, which occur at $x=\cos (\pi k / n)$ where $k$ is odd (in Figure 1 these were indicated by a solid vertical line). But there are also such lines of fixed points vertical at $x=\cos (\pi k / n)$ with $k$ even (in Figure 1 these were indicated by a dashed vertical line). The fractions $k / n$ with $k$ even fit in the (left side of the) ordered list (3.1) as follows:

$$
\frac{n-2 k}{n+2}>\frac{n-2 k-2}{n}>\frac{n-2 k-4}{n-2} \text {. }
$$

All of this can be seen in Figure 1.

We have indicated the existence of various curves of fixed points of $\alpha_{n}$ that lie in $\mathcal{T}_{+}$. They either have

(i) $\quad x=\cos (2 \pi k / n), k=1, \ldots, n / 2-1$,

or satisfy

(ii) $\quad z=x\left(1+U_{n-2}(x)\right) / U_{n-1}(x)$.

LEMMA 3.6. Let $n \in 4 \mathbb{N}$.

(a) Any fixed point of $\alpha_{n}$ in $\mathcal{T}_{+} \backslash V$ is of the form (i) or (ii) above.

(b) The reversing fixed points for $\alpha_{n}$ on $\partial \mathcal{T}$ are of the form

$$
\Pi\left(\begin{array}{c}
(-2 i-n j) /\left(4-n^{2}\right) \\
(n i+2 j) /\left(4-n^{2}\right)
\end{array}\right),
$$

for integers $i, j$. The reversing points form a lattice with generators

$$
v_{1}=\left(\begin{array}{c}
1 /(n+2) \\
1 /(n+2)
\end{array}\right), \quad v_{2}=\left(\begin{array}{c}
2 /\left(4-n^{2}\right) \\
-n /\left(4-n^{2}\right)
\end{array}\right) .
$$

(c) The preserving fixed points on $\partial \mathcal{T}$ are of the form

$$
\Pi\left(\begin{array}{l}
i / n \\
j / n
\end{array}\right), \quad \text { for integers } i, j .
$$


Proof. Suppose that $(x, x, z) \in \partial \mathcal{T} \backslash V$ is a fixed point for $\alpha_{n}$. If $x \neq \cos (2 \pi k / n)$ for some $k \in\{1, \ldots, n / 2-1\}$, then we have a fixed point of type (i). If not, then $U_{n-1}(x) \neq 0$ and we see that $z=\left(1+U_{n-2}(x)\right) / U_{n-1}(x)$ from Corollary 2.2. This does (a).

For (b), we note that from Lemma 2.9 we have

$$
\Phi\left(\alpha_{n}\right)=\left(\begin{array}{cc}
1 & n \\
-n & 1-n^{2}
\end{array}\right)
$$

Thus we need to solve

$$
\left(\begin{array}{cc}
1 & n \\
-n & 1-n^{2}
\end{array}\right) v=-v \quad \bmod \mathbb{Z}^{2}
$$

which is

$$
\left(\begin{array}{cc}
2 & n \\
-n & 2-n^{2}
\end{array}\right) v=\left(\begin{array}{l}
i \\
j
\end{array}\right) \in \mathbb{Z}^{2}
$$

Thus any reversing fixed point $v$ has the form

$$
\begin{aligned}
v & =\left(\begin{array}{cc}
2 & n \\
-n & 2-n^{2}
\end{array}\right)^{-1}\left(\begin{array}{l}
i \\
j
\end{array}\right)=\frac{1}{4-n^{2}}\left(\begin{array}{cc}
2-n^{2} & -n \\
n & 2
\end{array}\right)\left(\begin{array}{l}
i \\
j
\end{array}\right) \\
& =\left(\begin{array}{c}
\left(2-n^{2}\right) i /\left(4-n^{2}\right)+(-n j) /\left(4-n^{2}\right) \\
n i /\left(4-n^{2}\right)+2 j /\left(4-n^{2}\right)
\end{array}\right)=\left(\begin{array}{c}
i+(-2 i-n j) /\left(4-n^{2}\right) \\
(n i+2 j) /\left(4-n^{2}\right)
\end{array}\right),
\end{aligned}
$$

and any point of this form is a reversing fixed point.

The reversing points clearly form a lattice, which we denote by $\mathfrak{L}_{-}$. To see that $v_{1}, v_{2}$ are a basis for $\mathfrak{L}_{-}$we first note that they are both in $\mathfrak{L}_{-}$: for $v_{1}$ put $i=1, j=1-n$; for $v_{2}$ put $i=1, j=-n$. Now to see that these generate $\mathfrak{L}_{-}$we need only note that

$$
\operatorname{det}\left(\begin{array}{cc}
1 /(n+2) & 2 /\left(n^{2}-4\right) \\
1 /(n+2) & -n /\left(n^{2}-4\right)
\end{array}\right)=1 /\left(4-n^{2}\right),
$$

which is the determinant of

$$
\left(\begin{array}{cc}
2 & n \\
-n & 2-n^{2}
\end{array}\right)^{-1}
$$

(c) We have already seen in Lemma 2.3 that any point of the form $\Pi(i / n, j / n)^{\mathrm{T}}$ is a preserving fixed point. An argument similar to (b) above shows the converse.

Proposition 3.7. Let $n$ be even, let $k$ be even and let $x=\cos (k \pi / n)$. Then $(x, x, z)^{\mathrm{T}}$ is a curve of fixed points for $\alpha_{n}$. The point $\left(x, x, x^{2}\right)^{\mathrm{T}}$ is also on the curve of fixed points $\left(x, x, x\left(1+U_{n-2}(x)\right) / U_{n-1}(x)\right)^{\mathrm{T}}$.

Proof. We have noted above that $(x, x, z)^{\mathrm{T}}$ is a curve of fixed points for $\alpha_{n}$. To show that $\left(x, x, x^{2}\right)^{\mathrm{T}}$ is on the curve of fixed points $\left(x, x, x\left(1+U_{n-2}(x)\right) / U_{n-1}(x)\right)^{\mathrm{T}}$ we need to show that $x\left(1+U_{n-2}(x)\right) / U_{n-1}(x)=x^{2}$. Since $k$ is even we have $1+U_{n-2}(x)=$ $U_{n-1}(x)=0$ and so by L'Hôpital's rule we need to show that $U_{n-2}^{\prime}(x) / U_{n-1}^{\prime}(x)=x$ or, equivalently, that $U_{n-2}^{\prime}(x)-x U_{n-1}^{\prime}(x)=0$.

But differentiating $U_{n-1}(x)^{2}-2 x U_{n-2}(x) U_{n-1}(x)+U_{n-2}(x)^{2}=1$ from Proposition 2.6 (vii) gives $-2 x U_{n-2}(x) U_{n-1}^{\prime}(x)+2 U_{n-2}(x) U_{n-2}^{\prime}(x)=0$, on putting $U_{n-1}(x)=0$, as required.

Question. Can a symbolic dynamical system be developed for $\alpha_{n} \mid \partial \mathcal{T}$ using $\alpha_{n}\left(\mathcal{T}_{ \pm}\right)$that sheds light on the dynamics of $\alpha_{n} \mid \mathcal{T}$ at different levels? Compare Figure 3 in $\S 6$. 
4. The type (F3) and type (F4) cases

We now determine the polynomial $K_{n}(x, y)$ which defines the curves of fixed points of types (F3) and (F4).

From Corollary 2.2 we see that if $(x, y, z)^{\mathrm{T}}$ is a fixed point for $\alpha_{n}$ where $U_{n-1}(y) \neq 0$, then $z=x\left(1+U_{n-2}(y)\right) / U_{n-1}(y)$. Further, using the terminology of that result, we have $x^{*}=x$ and

$$
\begin{aligned}
U_{n}\left(x^{*}\right) y-U_{n-1}\left(x^{*}\right)\left[-x U_{n-1}(y)+z U_{n}(y)\right] & =y, \\
U_{n-1}\left(x^{*}\right) y-U_{n-2}\left(x^{*}\right)\left[-x U_{n-1}(y)+z U_{n}(y)\right] & =z .
\end{aligned}
$$

Substituting for $z$ and clearing the denominators of these equations gives two polynomials $q_{2}(x, y)=q_{2, n}(x, y)$ and $q_{3}(x, y)=q_{3, n}(x, y)$ :

$$
\begin{aligned}
q_{2}(x, y)= & U_{n-1}(x) U_{n-1}(y)^{2} x+U_{n}(x) y U_{n-1}(y)-U_{n-1}(x) U_{n}(y) x \\
& -U_{n-1}(x) U_{n}(y) x U_{n-2}(y)-y U_{n-1}(y) \\
q_{3}(x, y)= & U_{n-2}(x) U_{n-1}(y)^{2} x+U_{n-1}(x) y U_{n-1}(y)-U_{n-2}(x) U_{n}(y) x \\
& -U_{n-2}(x) U_{n}(y) x U_{n-2}(y)-x-U_{n-2}(y) x .
\end{aligned}
$$

Thus in looking for fixed points for $\alpha_{n}$ we are looking for simultaneous solution of $q_{2}(x, y)$ and $q_{3}(x, y)$. Thus finding $\operatorname{gcd}\left(q_{2}, q_{3}\right)$ seems necessary.

PROPOSITION 4.1. Let $n=2 m>2$. Then the greatest common divisor of $q_{2}(x, y)$ and $q_{3}(x, y)$ is $g=g_{n}(x, y)$ where

$$
\begin{aligned}
g= & -x^{2} U_{m-1}(x) U_{m-2}(y)^{2}-y^{2} U_{m-2}(y)^{2} x U_{m-2}(x)+x^{2} U_{m-1}(x) y U_{m-1}(y) U_{m-2}(y) \\
& +U_{m-1}(x) y^{2} U_{m-2}(y)^{2}-U_{m-1}(x) y^{2}+2 y^{3} U_{m-1}(y) U_{m-2}(y) x U_{m-2}(x) \\
& -2 U_{m-1}(x) y^{3} U_{m-1}(y) U_{m-2}(y)-2 x y U_{m-1}(y) U_{m-2}(y) U_{m-2}(x)+y^{2} x U_{m-2}(x) \\
& +x^{2} U_{m-1}(x)+x U_{m-2}(y)^{2} U_{m-2}(x)+y U_{m-1}(x) U_{m-1}(y) U_{m-2}(y)-x U_{m-2}(x) .
\end{aligned}
$$

Further, we have $q_{3}(x, y)=-4 U_{m-2}(x) g$ and $q_{2}(x, y)=4 U_{m-1}(x) g$.

Lastly, $g(x, y)$ is divisible by $x^{2}-y^{2}$ and by $U_{m-1}(y): g_{n}(x, y)=U_{m-1}(y)$ $J_{n}(x, y)$ where

$$
\begin{aligned}
J_{n}(x, y)= & U_{m-1}(y) U_{m-2}(x)\left(y^{2}-1\right) x+U_{m-1}(y) U_{m-1}(x)\left(x^{2}-y^{2}\right) \\
& +U_{m-1}(x) U_{m-2}(y)\left(1-x^{2}\right) y .
\end{aligned}
$$

Proof. First consider the polynomial $q_{3}(x, y)$ which involves $U_{n-2}(x)$ and $U_{n-2}(y)$. Substitute $U_{n-2}(x)=2 x U_{n-1}(x)-U_{n}(x)$ and $U_{n-2}(y)=2 y U_{n-1}(y)-U_{n}(y)$ so that now $q_{3}(x, y)$ only involves $U_{n}(x), U_{n}(y), U_{n-1}(x)$ and $U_{n-1}(y)$ (as well as $x, y$ ). Now use Proposition 2.6(viii) and (ix):

$$
U_{n}=U_{2 m}=U_{m}^{2}-U_{m-1}^{2}, \quad U_{n-1}=U_{2 m-1}=2 U_{m} U_{m-1}-2 x U_{m-1}^{2}
$$

to reduce $q_{3}(x, y)$ to a polynomial in $U_{m}(x), U_{m-1}(x), U_{m}(y), U_{m-1}(y)$ and $x, y$. Now substitute $U_{m}(x)=2 x U_{m-1}(x)-U_{m-2}(x)$ and $U_{m}(y)=2 y U_{m-1}(y)-U_{m-2}(y)$ and use the relation

$$
U_{m-1}(x)^{2}=1+2 x U_{m-1}(x) U_{m-2}(x)-U_{m-2}(x)^{2}
$$


from Proposition 2.6 to get rid of powers of $U_{m-1}(x)$ of exponent greater than one. Lastly, use

$$
U_{m-1}(y)^{2}=1+2 y U_{m-1}(y) U_{m-2}(y)-U_{m-2}(y)^{2}
$$

to get rid of powers of $U_{m-1}(y)$ of exponent greater than one. What one now has is $4 U_{m-2}(x) g$. This does $q_{3}(x, y)$.

For $q_{2}(x, y)$ : Substitute $U_{n-2}(x)=2 x U_{n-1}(x)-U_{n}(x)$ and $U_{n-2}(y)=2 y U_{n-1}(y)-$ $U_{n}(y)$ so that now $q_{2}(x, y)$ only involves $U_{n}(x), U_{n}(y), U_{n-1}(x), U_{n-1}(y)$ and $x, y$. Again use Proposition 2.6(viii) and (ix) to reduce $q_{2}(x, y)$ to a polynomial in $U_{m}, U_{m-1}$ and $x, y$. Now use

$$
U_{m-1}(x)^{2}=1+2 x U_{m-1}(x) U_{m-2}(x)-U_{m-2}(x)^{2}
$$

(and the corresponding $y$ equation) and we obtain $q_{2}(x, y)=-2 U_{m-1}(x) g_{*}$. One now shows that $g_{*}=-2 g$ by substituting

$$
U_{m}(x)=2 x U_{m-1}(x)-U_{m-2}(x), \quad U_{m}(y)=2 y U_{m-1}(y)-U_{m-2}(y)
$$

and

$$
U_{m-2}(y)^{2}=1+2 y U_{m-1}(y) U_{m-2}(y)-U_{m-2}(y)^{2}
$$

in $g_{*}+2 g$ to get 0 . This does $q_{2}(x, y)$.

Now if $y=x$, then Lemma 3.2 shows that $\left(x, x, x\left(1+U_{n-2}(x)\right) / U_{n-1}(x)\right)^{\mathrm{T}}$ is a curve of fixed points. It follows that $q_{2}(x, x)=q_{3}(x, x)=0$. Since $U_{m-2}(x)$ and $U_{m-1}(x)$ do not share a root (see (2.2)) it follows that $g(x, x)=0$ and so $x-y$ is a factor of $g_{n}(x, y)$. Similarly, since $n$ is even, we see that $x+y$ is a factor of $g_{n}(x, y)$. Thus $(x-y)(x+y)$ divides $g_{n}(x, y)$.

By collecting all terms of $g_{n}$ that contain $U_{m-1}(y)$ we can write

$$
\begin{aligned}
g_{n}(x, y)= & U_{m-1}(y) H_{n}(x, y) \\
& +\left(1-U_{m-2}(y)^{2}\right)\left(y^{2} x U_{m-2}(x)+x^{2} U_{m-1}(x)-y^{2} U_{m-1}(x)-x U_{m-2}(x)\right)
\end{aligned}
$$

for some polynomial $H_{n}(x, y)$. By adding a suitable multiple of $U_{m-1}(y)$ we can change $H_{n}(x, y)$ to a polynomial $J_{n}(x, y)$ so that

$$
\begin{aligned}
g_{n}(x, y)= & U_{m-1}(y) J_{n}(x, y) \\
+ & \left(1-U_{m-2}(y)^{2}-U_{m-1}(y)^{2}+2 y U_{m-1}(y) U_{m-2}(y)\right) \\
& \quad \times\left(y^{2} x U_{m-2}(x)+x^{2} U_{m-1}(x)-y^{2} U_{m-1}(x)-x U_{m-2}(x)\right) \\
= & U_{m-1}(y) J_{n}(x, y),
\end{aligned}
$$

the last equality coming from Proposition 2.6(vii). Thus $U_{m-1}(y)$ divides $g_{2 m}(x, y)$.

Carrying out the calculation indicated in this last part of the proof of Proposition 4.1 gives the expression (4.1) for the polynomial $J_{n}(x, y)$.

Proposition 4.1 shows that $x^{2}-y^{2}$ divides $J_{n}(x, y)$ and one easily checks that $J_{n}(y, x)=-J_{n}(x, y)$. Since $U_{k}(0)=0$ for $k$ odd it easily follows (in the case where $m$ is even) from (4.1) that $x y$ is also a factor of $J_{n}(x, y)$. We thus define the polynomial $K_{n}(x, y)$ by

$$
K_{n}(x, y)=J_{n}(x, y) /\left(x y\left(x^{2}-y^{2}\right)\right) .
$$


Example 4.2. For $n=8,12$ we have

$$
\begin{aligned}
g_{8} & =16 y^{2} x\left(2 y^{2}-1\right)(x-y)(x+y)\left(8 y^{2} x^{2}-6 x^{2}+5-6 y^{2}\right), \\
K_{8}(x, y) & =8 y^{2} x^{2}-6 x^{2}+5-6 y^{2}
\end{aligned}
$$

and

$$
\begin{aligned}
g_{12}= & 4 y^{2} x(2 y-1)(2 y+1)\left(4 y^{2}-3\right)(x+y)(x-y) \\
& \times\left(256 x^{4} y^{4}-320 x^{4} y^{2}+80 x^{4}+416 y^{2} x^{2}-112 x^{2}-320 y^{4} x^{2}\right. \\
& \left.\quad+80 y^{4}+35-112 y^{2}\right), \\
K_{12}(x)= & 256 x^{4} y^{4}-320 x^{4} y^{2}+80 x^{4}+416 y^{2} x^{2}-112 x^{2}-320 y^{4} x^{2} \\
& +80 y^{4}+35-112 y^{2} .
\end{aligned}
$$

Suppose that we have a function $y=y(x)$ which satisfies $K_{n}(x, y(x))=0$. Then we certainly have $q_{2}(x, y(x))=q_{3}(x, y(x))=0$. Thus

$$
\gamma(x)=\left(x, y(x), x\left(1+U_{n-2}(y(x))\right) / U_{n-1}(y(x))\right)
$$

will give a curve of fixed points for $\alpha_{n}$. We will say that such a curve is of type (F3) in our classification scheme if it does not meet the planes $x= \pm y$ and will be of type (F4) if it does.

Example 4.2 (continued). For $n=8$ we have $K_{8}(x, y)=8 y^{2} x^{2}-6 x^{2}+5-6 y^{2}$ and we can solve this equation for $y$ to obtain

$$
y(x)= \pm \sqrt{\frac{6 x^{2}-5}{2\left(4 x^{2}-3\right)}} .
$$

For $n=12$ we get a degree four equation in $y$ which we solve to give the following four solutions:

$$
y(x)=\frac{ \pm \sqrt{\left(16 x^{4}-20 x^{2}+5\right)\left(40 x^{4}-52 x^{2}+14 \pm \sqrt{320 x^{8}-768 x^{6}+624 x^{4}-196 x^{2}+21}\right)}}{2\left(16 x^{4}-20 x^{2}+5\right)} .
$$

One can solve $K_{n}(x, y)=0$ for $y$ and solve this using only radicals for $n=4,8$, 12, 16, 20 (when $K_{n}(x, y)$ is irreducible). Thus the Galois group of the polynomial $K_{n}(x, y)$, when considered as an element of the polynomial ring $\mathbb{Q}(x)[y]$, is solvable for $n=4,8,12,16,20$. This is not the case for $n=24$ however.

\section{CONJECTURES 4.3.}

(i) Let $n=4 m$. Then $K_{n}(x, y)$ is irreducible.

(ii) The genus of $K_{4 m}(x, y)$ is $(2 m-3)^{2}$.

Using Magma [BC] we have checked these conjectures for $4 m \leq 40$.

Proposition 4.4. Let $n=2 m$ and $\theta=\left(\theta_{1}, \theta_{2}\right)^{\mathrm{T}}$ be such that $\Pi(\theta)=(x, y, z)^{\mathrm{T}}$ is $a$ fixed point of $\alpha_{n}$. Then $J_{n}(x, y)=0$. 
Proof. The point $\Pi(\theta)$ is either a preserving or a reversing fixed point and so $\theta$ is given by Lemma 3.6(b) and (c). Suppose first that $\pi(\theta)$ is a preserving fixed point. Then by that same result we may take $\theta=(i / n, j / n)$ so that $x=\cos (2 \pi i / n), y=\cos (2 \pi j / n)$. We may clearly assume that $0<i, j \leq n$. From (2.2) we see that $U_{m-1}(\cos (2 \pi k / n))=0$ unless $k \equiv 0 \bmod m$. Now if $\{i, j\} \cap\{m, 2 m\}=\emptyset$, then by the above $U_{m-1}(x)=$ $U_{m-1}(y)=0$, which gives $J_{n}(x, y)=0+0+0$, as required.

So now assume that $i \in\{m, 2 m\}, j \notin\{m, 2 m\}$. Then $x^{2}=\cos (2 \pi i / n)^{2}=1$ and $U_{m-1}(y)=0$; it follows that $J_{n}(x, y)=0$. The case $i \notin\{m, 2 m\}, j \in\{m, 2 m\}$ is similar. If $i, j \in\{m, 2 m\}$, then $x, y= \pm 1$, so that $x^{2}=y^{2}=1$ and it follows that $J_{n}(x, y)=0$. This concludes the preserving case.

So now assume that $(x, y, z)^{\mathrm{T}}$ is a reversing fixed point. Here $\theta$ is given by Lemma 3.6(b):

$$
\theta=\left(\begin{array}{c}
\theta_{1} \\
\theta_{2}
\end{array}\right)=\left(\begin{array}{c}
\left(2-n^{2}\right) i /\left(4-n^{2}\right)+(-n j) /\left(4-n^{2}\right) \\
n i /\left(4-n^{2}\right)+2 j /\left(4-n^{2}\right)
\end{array}\right) .
$$

Now $x=\cos \left(2 \pi \theta_{1}\right), y=\cos \left(2 \pi \theta_{2}\right)$ and using (2.2) we see that

$$
U_{m-2}(x)=\frac{\sin \left(2 \pi \theta_{1}(m-1)\right)}{\sin \left(2 \pi \theta_{1}\right)}=\frac{\sin \left(2 \pi \theta_{1} m\right) \cos \left(2 \pi \theta_{1}\right)-\cos \left(2 \pi \theta_{1} m\right) \sin \left(2 \pi \theta_{1}\right)}{\sin \left(2 \pi \theta_{1}\right)},
$$

with a similar expression for $U_{m-2}(y)$. Substituting these expressions into (4.1) we obtain an expression whose numerator is

$$
\begin{aligned}
J_{n}(x, y)= & -\sin \left(2 \pi \theta_{2} m\right) \cos \left(2 \pi \theta_{1}\right) \cos \left(2 \pi \theta_{1} m\right) \sin \left(2 \pi \theta_{1}\right) \\
& +\sin \left(2 \pi \theta_{2} m\right) \cos \left(2 \pi \theta_{1}\right) \cos ^{2}\left(2 \pi \theta_{2}\right) \cos \left(2 \pi \theta_{1} m\right) \sin \left(2 \pi \theta_{1}\right) \\
& +\sin \left(2 \pi \theta_{1} m\right) \cos \left(2 \pi \theta_{2}\right) \cos \left(2 \pi \theta_{2} m\right) \sin \left(2 \pi \theta_{2}\right) \\
& -\sin \left(2 \pi \theta_{1} m\right) \cos \left(2 \pi \theta_{2}\right) \cos ^{2}\left(2 \pi \theta_{1}\right) \cos \left(2 \pi \theta_{2} m\right) \sin \left(2 \pi \theta_{2}\right) .
\end{aligned}
$$

Now using $\cos ^{2}\left(2 \pi \theta_{k}\right)=1-\sin ^{2}\left(2 \pi \theta_{k}\right), k=1,2$, we substitute for $\cos ^{2}\left(2 \pi \theta_{k}\right)$ and find that this expression factors:

$$
\begin{aligned}
\sin \left(2 \pi \theta_{1}\right) \sin \left(2 \pi \theta_{2}\right) \times & \left(-\sin \left(2 \pi \theta_{2} m\right) \sin \left(2 \pi \theta_{2}\right) \cos \left(2 \pi \theta_{1}\right) \cos \left(2 \pi \theta_{1} m\right)\right. \\
+ & \sin \left(2 \pi \theta_{1} m\right) \sin \left(2 \pi \theta_{1}\right) \cos \left(2 \pi \theta_{2}\right) \cos \left(2 \pi \theta_{2} m\right) .
\end{aligned}
$$

We will show that the last factor of (4.3) is zero for integral $i, j, m$.

Now use the fact that

$$
\cos \left(2 \pi \theta_{k}(m+1)\right)=\cos \left(2 \pi \theta_{k} m\right) \cos \left(2 \pi \theta_{k}\right)-\sin \left(2 \pi \theta_{k} m\right) \sin \left(2 \pi \theta_{k}\right)
$$

to substitute for the sines in the last factor of the expression (4.3). There is cancelling and we obtain

$$
\cos \left(2 \pi \theta_{1}\right) \cos \left(2 \pi \theta_{1} m\right) \cos \left(2 \pi \theta_{2}(m+1)\right)-\cos \left(2 \pi \theta_{2}\right) \cos \left(2 \pi \theta_{2} m\right) \cos \left(2 \pi \theta_{1}(m+1)\right) .
$$

We now note that $2 \pi \theta_{2} m=\pi i-2 \pi \theta_{1}, 2 \pi \theta_{1} m=(2 m i+j) \pi-2 \pi \theta_{2}$. Substituting, and noting that we can ignore integral multiples of $2 \pi$, we obtain

$$
\begin{aligned}
& \cos \left(2 \pi \theta_{1}\right) \cos \left(j \pi-2 \pi \theta_{2}\right) \cos \left(2 \pi \theta_{2}+\pi i-2 \pi \theta_{1}\right) \\
& \quad-\cos \left(2 \pi \theta_{2}\right) \cos \left(\pi i-2 \pi \theta_{1}\right) \cos \left(2 \pi \theta_{1}+j \pi-2 \pi \theta_{2}\right) .
\end{aligned}
$$


Now one checks that for integral $i, j$ this expression is always 0 , there being four cases depending on the parity of $i, j$. For example, if $i, j$ are both even, then (4.4) is

$\cos \left(2 \pi \theta_{1}\right) \cos \left(2 \pi \theta_{2}\right) \cos \left(2 \pi \theta_{2}-2 \pi \theta_{1}\right)-\cos \left(2 \pi \theta_{2}\right) \cos \left(-2 \pi \theta_{1}\right) \cos \left(2 \pi \theta_{1}-2 \pi \theta_{2}\right)=0$.

This concludes the proof.

LEMMA 4.5. There are no points and curves of type $(F 4)$ if $K_{n}(x, x)$ has no real solutions.

Proof. If we have a curve of type (F4), then there is a point $\left(x_{0}, x_{0}, z_{0}\right)^{\mathrm{T}} \in \mathcal{T}_{+}$which is a limit point of fixed points $\left(x_{i}, y_{i}, z_{i}\right)^{\mathrm{T}}, i \in \mathbb{N}$, where $x_{i} \neq y_{i}$ so that the points $\left(x_{i}, y_{i}\right)^{\mathrm{T}}$ are solutions to the equation $K_{n}(x, y)=0$. We thus have $K_{n}\left(x_{0}, x_{0}\right)=0$ where $x_{0} \in \mathbb{R}$.

We now give another expression for $K_{4 m}(x, y)$.

PROPOSITION 4.6.

(i) For all $m \in \mathbb{N}$ we have

$$
J_{4 m+4}(x, y)-J_{4 m}(x, y)=2\left(x^{2}-y^{2}\right) T_{2 m+1}(x) T_{2 m+1}(y) .
$$

(ii) For all $m \in \mathbb{N}$ and $x, y \in \mathbb{R}$, we have

$$
x y K_{4 m}(x, y)=2 \sum_{i=1}^{m} T_{2 i-1}(x) T_{2 i-1}(y) .
$$

(iii) For all $m \in \mathbb{N}$ the polynomial $K_{4 m}(x, y)$ has degree $4 m-4$. As a polynomial in $x$ (or $y) K_{4 m}(x, y)$ has degree $2 m-2$.

(iv) For all $m \in \mathbb{N}$ we have $K_{4 m}(x, y)=K_{4 m}(y, x)=K_{4 m}(-x, y)=K_{4 m}(x,-y)$.

Proof. (i) $J_{4 m}(x, y)$ is given in (4.1), from which we also obtain an expression for $J_{4 m+4}(x, y)$ :

$$
\begin{aligned}
J_{4 m}(x, y)= & U_{2 m-1}(y) U_{2 m-2}(x)\left(y^{2}-1\right) x+U_{2 m-1}(y) U_{2 m-1}(x)\left(x^{2}-y^{2}\right) \\
& +U_{2 m-1}(x) U_{2 m-2}(y)\left(1-x^{2}\right) y, \\
J_{4 m+4}(x, y)= & U_{2 m+1}(y) U_{2 m}(x)\left(y^{2}-1\right) x+U_{2 m+1}(y) U_{2 m+1}(x)\left(x^{2}-y^{2}\right) \\
& +U_{2 m+1}(x) U_{2 m}(y)\left(1-x^{2}\right) y .
\end{aligned}
$$

Now $U_{2 m-2}(x)$ and $U_{2 m-2}(y)$ occur in (4.6) and we substitute $2 x U_{2 m-1}(x)-U_{2 m}(x)$ for $U_{2 m-2}(x)$ (and similarly for $\left.U_{2 m-2}(y)\right)$ to give an expression which we denote by $j_{4 m}(x, y)$. Similarly $U_{2 m+1}(x)$ and $U_{2 m+1}(y)$ occur in (4.7) and we substitute $2 x U_{2 m}(x)-$ $U_{2 m-1}(x)$ for these (and similarly for $\left.U_{2 m+1}(y)\right)$ to give an expression $j_{4 m+4}(x, y)$. Thus $j_{4 m+4}(x, y)$ and $j_{4 m}(x, y)$ are both polynomials in $x, y, U_{2 m}(x), U_{2 m}(y), U_{2 m-1}(x)$ and $U_{2 m-1}(y)$. Examining $j_{4 m+4}(x, y)-j_{4 m}(x, y)$ one sees that it factors as

$2\left(x^{2}-y^{2}\right)\left(x U_{2 m}(x)-U_{2 m-1}(x)\right)\left(y U_{2 m}(y)-U_{2 m-1}(y)\right)=2\left(x^{2}-y^{2}\right) T_{2 m+1}(x) T_{2 m+1}(y)$,

as required. Here we have used the fact [Ri, p. 9] that for any $n \geq 1$ we have $T_{n}(x)=U_{n}(x)-x U_{n-1}(x)=x U_{n-1}(x)-U_{n-2}(x)$.

(ii) Let $x \neq \pm y$. We note that (i) gives

$$
J_{4 m}(x, y)=2\left(x^{2}-y^{2}\right) \sum_{i=1}^{m} T_{2 i-1}(x) T_{2 i-1}(y) .
$$


But we also have the definition $J_{4 m}=\left(x^{2}-y^{2}\right) x y K_{4 m}(x, y)$; thus if $x \neq \pm y$, then (ii) follows immediately since we can divide by $x^{2}-y^{2}$. But any point $(x, x)$ or $(x,-x)$ is a limit point of the complement of the $x= \pm y$ planes and so (4.5) is true for all $x, y$ by continuity.

(iii) It is easy to see that $T_{k}(x)$ has degree $k$ and that $T_{2 i-1}(x) / x$ and $T_{2 i-1}(y) / y$ have degree $2 i-2$. Thus (iii) follows from (4.5).

(iv) It is clear from (4.5) that $K_{4 m}(x, y)=K_{4 m}(y, x)$. Now $T_{2 i-1}(x) / x$ and $\left.T_{2 i-1}(y) / y\right)$ are polynomials in $x^{2}$ and $y^{2}$ respectively, and so $K_{4 m}(-x, y)=$ $K_{4 m}(x, y)=K_{4 m}(x,-y)$ as required.

PROPOSITION 4.7. Let $n=4 m, m \in \mathbb{N}$. Then there are no type (F4) curves of fixed points in $\mathcal{T}$ for $\alpha_{n}$.

We use the following result.

LEMMA 4.8 .

(i) For all $m \in \mathbb{N}$ we have $x^{2} K_{4 m}(x, x)=2 \sum_{i=1}^{m} T_{2 i-1}^{2}(x)$.

(ii) For all $m \in \mathbb{N}$ we have $K_{4 m}(0,0)=2 \sum_{i=1}^{m}(2 i-1)^{2}$.

Proof. (i) This is immediate from (4.5).

For (ii) we note that $T_{2 i+1}(x)$ is divisible by $x$ and by induction we see that the coefficient of $x$ in $T_{2 i+1}(x)$ is $\pm(2 i+1)$. Thus the coefficient of $x^{2}$ in $T_{2 i+1}^{2}(x)$ is $(2 i+1)^{2}$ and (ii) follows from (i).

Proof of Proposition 4.7. By Lemma 4.5 it will suffice to show that $K_{4 m}(x, x) \neq 0$ for all $-1 \leq x \leq 1$. But from Lemma 4.8(i) we see that, if $K_{n}(x, x)=0$ with $x \neq 0$, then $x$ has to be a root of each of $T_{1}(x) / x, T_{3}(x) / x, \ldots, T_{2 m-1}(x) / x$. But each pair $T_{2 k+1}(x) / x, T_{2 k-1}(x) / x$ of polynomials is coprime (this follows from the fact [Ri, p. 1] that $\left.T_{n}(x)=\cos (n \arccos (x))\right)$ and so no such $x \neq 0$ is possible, leaving us to consider the case $x=0$.

Now Lemma 4.8(ii) clearly shows that $K_{4 m}(0,0) \neq 0$ and so Proposition 4.7 follows. $\square$

We now discuss the following consequences of (4.5) and the results of this section.

Now in $[-1,1]^{2}$ the sequence of polynomials $K_{4 m}(x, x)$ has the property that $K_{4 r}(a, a) \geq K_{4 s}(a, a)$ if and only if $r \geq s$, and the graphs of $K_{4 r}(x, x)$ and $K_{4 s}(x, x)$ only meet when $r=s+1$, where they meet tangentially in the $x=y$ plane since $K_{4 s+4}(x, x)-K_{4 s}(x, x)=2 T_{2 s-1}(x)^{2}$. We draw the slice through the $x=y$ plane of the set of graphs $K_{4 m}(x, y)$ in Figure 2, where $x \in[0.5,1]$.

\section{Elements of the type $\sigma_{1}^{r} \sigma_{2}^{s}$ and their associated polynomials}

Here we show how some of the above techniques apply to slightly more general elements of $B_{3}$.

Fix $r, s \in \mathbb{N}$ where $|2-r s|>2$, and consider $\alpha_{r, s}=\sigma_{1}^{r} \sigma_{2}^{s}$. Then using Lemma 2.1 we see that

$$
\left(\begin{array}{l}
x \\
y \\
z
\end{array}\right) \alpha_{r, s}=\left(\begin{array}{c}
-x U_{s-2}(y)+z U_{s-1}(y) \\
U_{r}\left(x^{*}\right) y-U_{r-1}\left(x^{*}\right)\left[-x U_{s-1}(y)+z U_{s}(y)\right] \\
U_{r-1}\left(x^{*}\right) y-U_{r-2}\left(x^{*}\right)\left[-x U_{s-1}(y)+z U_{s}(y)\right]
\end{array}\right) .
$$




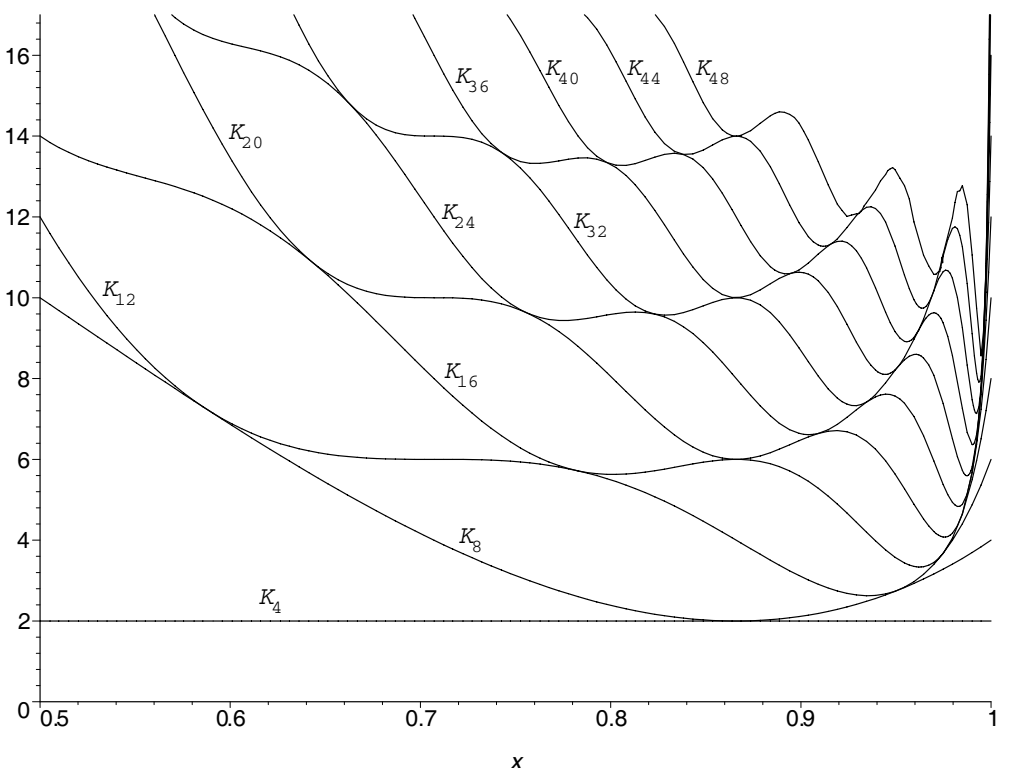

FIGURE 2. Graphs of some functions $F_{m}(x, x)$ for the study of curves of type (F4).

Here $x^{*}=-x U_{s-2}(y)+z U_{s-1}(y)$. As usual we are interested in the fixed points and curves of fixed points of $\alpha_{r, s}$ and so in particular we must have $x^{*}=x$. We can solve this for $z: z=x\left(1+U_{s-2}(y)\right) / U_{s-1}(y)$. As in the discussion at the beginning of $\S 4$ we also obtain (from equating the $y$ and $z$ expressions) two polynomials $q_{2, r, s}(x, y)$ and $q_{3, r, s}(x, y)$ :

$$
\begin{aligned}
q_{2, r, s}(x, y)= & U_{s-1}(y) U_{r}(x) y+x U_{r-1}(x) U_{s-1}^{2}(y) \\
& -x U_{r-1}(x)\left(1+U_{s-2}(y)\right) U_{s}(y)-y U_{s-1}(y), \\
q_{3, r, s}(x, y)= & y U_{r-1}(x) U_{s-1}(y)+x U_{s-1}^{2}(y) U_{r-2}(x) \\
& -x U_{s}(y) U_{r-2}(x)\left(1+U_{s-2}(y)\right)-x\left(1+U_{s-2}(y)\right) .
\end{aligned}
$$

The polynomials $q_{2, r, s}$ and $q_{3, r, s}$ have degree at most $2 s+r-2$ and they have the property that if $(a, b, c)$ is a fixed point of $\alpha_{r, s}$, then $q_{2, r, s}(a, b)=0, q_{3, r, s}(a, b)=0$. Let $g_{r, s}(x, y)=\operatorname{gcd}\left(q_{2, r, s}(x, y), q_{3, r, s}(x, y)\right)$.

Now note that the condition $|2-r s|>2$ guarantees that $\left|\operatorname{trace}\left(\Phi\left(\alpha_{r, s}\right)\right)\right|>2$, so that $\Phi\left(\alpha_{r, s}\right)$ is a hyperbolic matrix. Thus if $p=(a, b, c)^{\mathrm{T}} \in \partial \mathcal{T} \backslash V$ is a fixed point, then there is a curve of fixed points $\gamma(t)=\left(\gamma_{1}(t), \gamma_{2}(t), \gamma_{3}(t)\right)^{\mathrm{T}}$ through $p$ and we must have $g_{r, s}\left(\gamma_{1}(t), \gamma_{2}(t)\right)=0$. In particular we have $g_{r, s}(a, b)=0$.

Now note that the fixed points of $\alpha_{r, s}$ come in the reversing and preserving types, each of which gives a lattice in the torus $\mathbb{T}^{2}$. Let

$$
M=\Phi\left(\alpha_{r, s}\right)=\left(\begin{array}{cc}
1 & s \\
-r & 1-r s
\end{array}\right)
$$


Then the lattice $\mathcal{L}_{+}$of preserving fixed points for $M$ is $\left(M-I_{2}\right)^{-1}\left(\mathbb{Z}^{2}\right)$, while the lattice $\mathcal{L}_{-}$of reversing fixed points is $\left(M+I_{2}\right)^{-1}\left(\mathbb{Z}^{2}\right)$. Here

$$
\left(M-I_{2}\right)^{-1}=\left(\begin{array}{cc}
-1 & -1 / r \\
1 / s & 0
\end{array}\right) \quad\left(M+I_{2}\right)^{-1}=\left(\begin{array}{cc}
(2-r s) /(4-r s) & -s /(4-r s) \\
r /(4-r s) & 2 /(4-r s)
\end{array}\right) .
$$

The fixed points on $\partial \mathcal{T}$ for $\alpha_{r, s}$ are thus $\Pi\left(\mathcal{L}_{+} \cup \mathcal{L}_{-}\right)$. We also have

$$
\left|\mathcal{L}_{+}\right|=\left|\operatorname{det}\left(M-I_{2}\right)\right|=r s \quad \text { and } \quad\left|\mathcal{L}_{-}\right|=\left|\operatorname{det}\left(M+I_{2}\right)\right|=r s-4 .
$$

This discussion gives the following result.

\section{THEOREM 5.1.}

(i) Let $r, s \in \mathbb{N},|2-r s|>2$. Then there is a polynomial $g_{r, s}(x, y)$ of degree at most $2 s+r-2$ such that for any $p=(a, b, c)^{\mathrm{T}} \in \Pi\left(\mathcal{L}_{+} \cup \mathcal{L}_{-}\right)$we have $g_{r, s}(a, b)=0$.

(ii) If $\gamma(t)=\left(\gamma_{1}(t), \gamma_{2}(t), \gamma_{3}(t)\right)^{\mathrm{T}}$ is a curve of fixed points through $p$, then $g_{r, s}\left(\gamma_{1}(t), \gamma_{2}(t)\right)=0$.

\section{Dual sets of points for $\alpha_{n}$}

We study the curves $J_{n}(x, y)=0$ introduced in $\S 4$, considered as a subset of the $(x, y)$ plane $\mathbb{R}^{2} \times\{0\} \subset \mathbb{R}^{3}$. These curves contain the projections to the $(x, y)$-plane of all curves of fixed points of $\alpha_{n}$. Consider the intersection of the $J_{n}(x, y)=0$ curves with the lines $x=\cos (2 \pi i / n)$ or $y=\cos (2 \pi j / n)$ in the $(x, y)$-plane. See Figure 3 where we have drawn these curves for $n=20$; the intersections of these horizontal and vertical lines determine a vertical line in $\mathbb{R}^{3}$ containing the preserving fixed points as in Lemma 2.12.

The curves of $J_{n}(x, y)=0$ include the four lines $x=0, y=0, x= \pm y$; see Figure 3 again. In Figure 3 we have also indicated as small circles the images of the points of $\mathcal{L}_{-}$.

Let $\pi_{z}: \mathbb{R}^{3} \rightarrow \mathbb{R}^{2} \times\{0\} \subset \mathbb{R}^{3}$ be the projection onto the $(x, y)$-plane and let $\Pi_{z}=\pi_{z} \Pi: \mathbb{T}^{2} \rightarrow \mathbb{R}^{2} \times\{0\} \subset \mathbb{R}^{3}$.

Proposition 6.1. Fix $m, n \in \mathbb{N}$ with $n=2 m$ and $4 \mid n$, and consider $i, j \in\{0, \ldots, n\}$.

(1) For $i \in\{1, \ldots, n-1\}$ even we have $U_{n-1}(\cos (\pi i / n))=U_{m-1}(\cos (\pi i / n))=0$.

For $i \in\{1, \ldots, n-1\}$ odd we have $U_{n-1}(\cos (\pi i / n))=0 \neq U_{m-1}(\cos (\pi i / n))$.

(2) If $i, j \in\{0, \ldots, n\}$ are both even, then $J_{n}(\cos (\pi i / n), \cos (\pi j / n))=0$.

(3) If $i, j \in\{0, \ldots, n\}$ are both odd, then $J_{n}(\cos (\pi i / n), \cos (\pi j / n))=0$.

(4) If $i \in\{0, \ldots, n\} \backslash\{m\}$ is even and $J_{n}(\cos (\pi i / n), y)=0$, then $y=\cos (\pi j / n)$ for some even $j \in\{0, \ldots, n\}$. If $j \in\{0, \ldots, n\} \backslash\{m\}$ is even and $J_{n}(x, \cos (\pi j / n))=0$, then $x=\cos (\pi i / n)$ for some even $i \in\{0, \ldots, n\}$.

(5) If $i \in\{0, \ldots, n\}$ is odd and $J_{n}(\cos (\pi i / n), y)=0$, then $y=0$ or $\cos (\pi j / n)$ for some odd $j \in\{0, \ldots, n\}$. If $j \in\{0, \ldots, n\}$ is odd and $J_{n}(x, \cos (\pi j / n))=0$, then $x=0$ or $\cos (\pi i / n)$ for some odd $i \in\{0, \ldots, n\}$.

Proof. (1) These statements come from (2.2).

(2) Since $U_{m-1}(\cos (\pi i / n))=0$ or $U_{m-1}(\cos (\pi j / n))=0$ occurs in each of the terms of $J_{n}(\cos (\pi i / n), \cos (\pi j / n))$ in (4.1), this follows from (1) unless $\{i, j\} \cap\{0, n\} \neq \emptyset$. If $i \in\{0, n\}$ then $1-x^{2}=0$ and $J_{n}(x, y)=U_{m-1}(y)\left(y^{2}-1\right)\left(U_{m-2}(x) x-U_{m-1}(x)\right)=0$ for even $j$. 


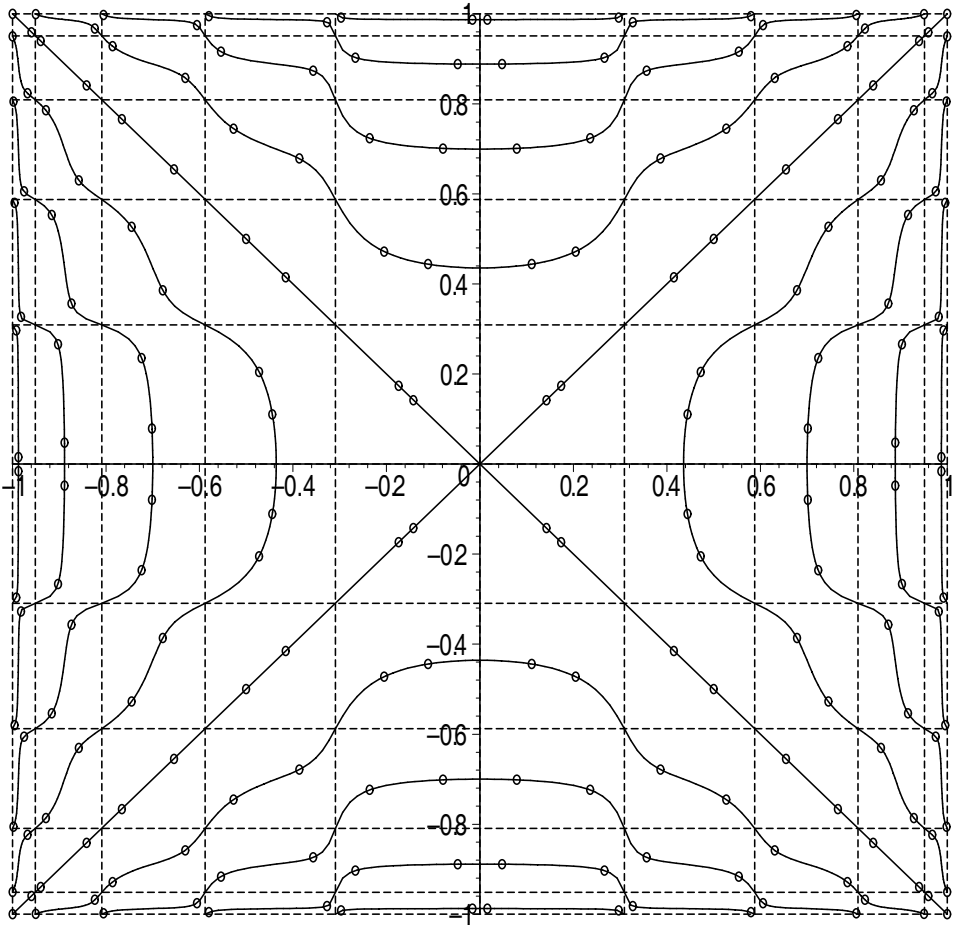

FIGURE 3. The projection to the $(x, y)$ plane of curves of fixed points of type (F2) an (F3).

(3) Using $U_{n-1}(\cos (\pi i / n))=U_{n-1}(\cos (\pi j / n))=0$ we see that $q_{2}(\cos (\pi i / n)$, $\cos (\pi j / n))=0$. Also

$$
\begin{aligned}
& q_{3}(\cos (\pi i / n), \cos (\pi j / n)) \\
& \quad=-x\left(U_{n-2}(\cos (\pi i / n)) U_{n}(\cos (\pi j / n))+1\right)\left(1+U_{n-2}(\cos (\pi j / n))\right)=0,
\end{aligned}
$$

since $U_{n-1}(\cos (\pi i / n))=1$ by Proposition $2.6(\mathrm{x})$ and $U_{n}(\cos (\pi j / n))=-1$ by Proposition 2.6(vi). Thus $g_{n}(\cos (\pi i / n), \cos (\pi j / n))=0$ and, since $U_{m-1}(\cos (\pi j / n)) \neq 0$, we have $J_{n}(\cos (\pi i / n), \cos (\pi j / n))=0$. Since, from (4.1), $J_{n}(y, x)=-J_{n}(x, y)$, the second statement follows from the first; this is also true for the other parts of this proposition.

(4) For $i \in\{1, \ldots, n-1\}$ even, $U_{m-1}(\cos (\pi i / n))=0$ and $U_{m-2}(\cos (\pi i / n)) \neq 0$ so $J_{n}(\cos (\pi i / n), y)=0$ implies that $U_{m-1}(y)\left(y^{2}-1\right) x=0$ and then either $x=0$ (which is excluded by $i \neq m)$ or $y= \pm 1$ or $y=\cos (\pi j / n)(0<j<n, j$ even $)$.

(5) For $i$ odd, $x=\cos (\pi i / n)$ satisfies $U_{2 m-1}(x)=0 \neq U_{m-1}(x)$, so by Proposition 2.6(ix) we have $U_{m}(x)=x U_{m-1}(x)$ and, from Proposition 2.6(vii) we have $U_{m-1}(x)^{2}-U_{m}(x)^{2}=1$. Substituting this in Proposition 2.6(vi) gives $U_{m-2}(x)=U_{m}(x)$. Now substituting $U_{m-2}(x)=x U_{m-1}(x)$ in (4.1) gives

$$
J_{n}(x, y)=U_{m-1}(x) U_{m-1}(y)\left[\left(y^{2}-1\right) x^{2}+\left(x^{2}-y^{2}\right)\right]+U_{m-1}(x) U_{m-2}(y)\left(1-x^{2}\right) y .
$$

Now $U_{m-1}(x)\left(x^{2}-1\right) \neq 0$, so $J_{n}(x, y)=0$ and $y \neq 0$ hold exactly at the roots 


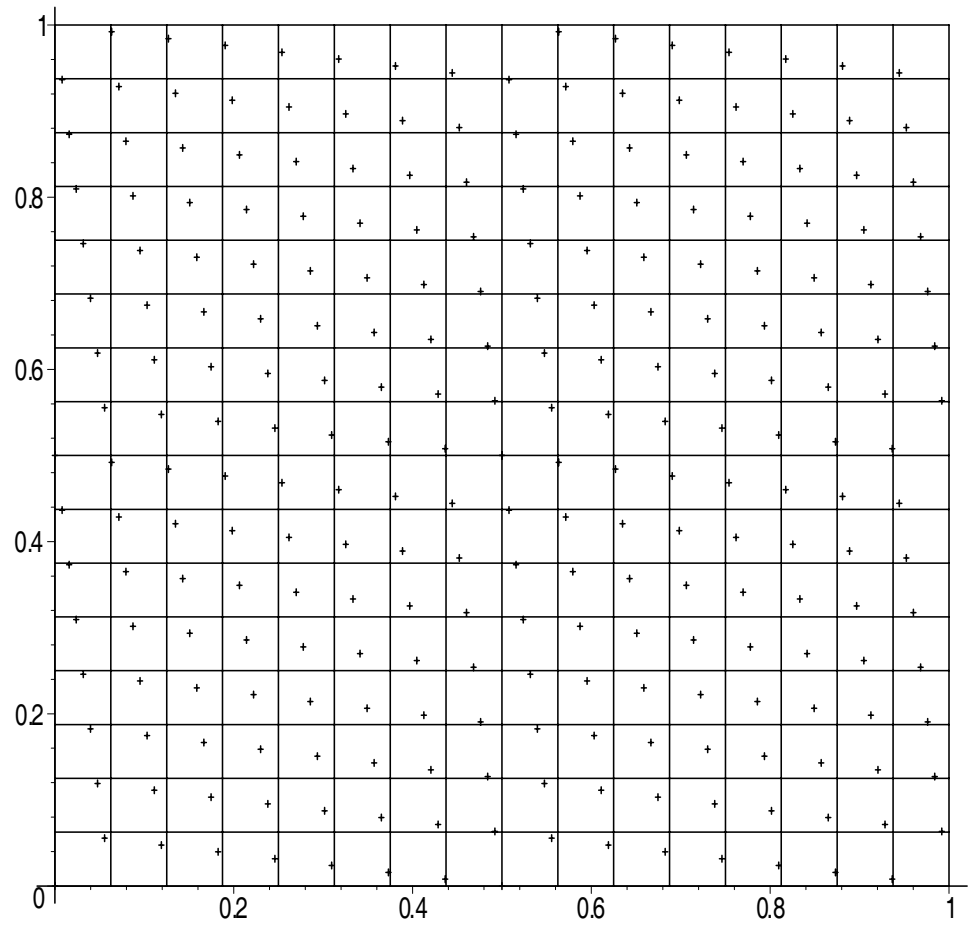

FIGURE 4. The lattice of reversing fixed points in $\mathbb{T}^{2}$.

of the polynomial $y U_{m-1}(y)-U_{m-2}(y)$ of degree $m$, and these are $\cos (\pi j / n)$ for odd $j \in\{0, \ldots, n\}$ by (3).

Recall that $\mathcal{L}_{-} \subset \mathbb{T}^{2}$ is the lattice of reversing points and that it is generated by the vectors $v_{1}, v_{2}$ given in Lemma 3.6. With the convention that we are drawing $\mathbb{T}^{2}$ as a square with sides identified we have drawn the points of $\mathcal{L}_{-}$in Figure 4 , for $n=16$, where these points are indicated by cross-hairs; we have also there indicated the preserving fixed points as the intersections of the horizontal and vertical lines.

The following result indicates the $\alpha_{n}$-duality.

THEOREM 6.2. Let $n$ be a multiple of 4 , put

$$
v_{1}=\left(\frac{1}{n+2}, \frac{1}{n+2}\right)^{\mathrm{T}}, \quad v_{2}=\left(\frac{-2}{n^{2}-4}, \frac{n}{n^{2}-4}\right)^{\mathrm{T}}
$$

and consider the lattice of reversing points spanned by $v_{1}, v_{2}$ as in Lemma 3.6.

(1) For $0<k \leq n / 4-2$ and $j=1, \ldots, n / 4-|k|-1$, the two reversing fixed points $\Pi\left((n / 4+1+k) v_{1}+(n / 4-k \pm j) v_{2}\right)$ are $\alpha_{n}$-dual by a curve of fixed points that meets the straight line of fixed points joining $\Pi\left((1 / 4+k / n, 1 / 2 \pm j / n)^{\mathrm{T}}\right)$.

(2) For $0<-k \leq n / 4-2$ and $j=1, \ldots, n / 4-|k|-1$, the two reversing fixed points $\Pi\left((n / 4+1+k) v_{1}+(n / 4-k-1 \pm j) v_{2}\right)$ are $\alpha_{n}$-dual by a curve of fixed points that meets the straight line of fixed points joining $\Pi\left((1 / 4+k / n, 1 / 2 \pm j / n)^{\mathrm{T}}\right)$. 
(3) For $j=1, \ldots, n / 4-1$, the two reversing points $\Pi\left((1 / 4,1 / 2)^{\mathrm{T}} \pm(j-1 / 2) v_{2}\right)$ are $\alpha_{n}$-dual by a curve of fixed points that meets the straight line of fixed points joining $\Pi\left((1 / 4,1 / 2)^{\mathrm{T}}\right)$ and $\Pi\left((1 / 4,0)^{\mathrm{T}}\right)$, which is the $y$-axis.

(4) For $0<k \leq n / 4-1$, the reversing point $\Pi\left((n / 4+1+k) v_{1}+(n / 4-k) v_{2}\right)$ is $\alpha_{n}$-dual to the preserving fixed point $\Pi\left((1 / 4+k / n, 1 / 2)^{\mathrm{T}}\right)$. For $0<-k \leq n / 4-1$, the reversing point $\Pi\left((n / 4+1+k) v_{1}+(n / 4-k-1) v_{2}\right)$ is $\alpha_{n}$-dual to the preserving fixed point $\Pi\left((1 / 4+k / n, 1 / 2)^{\mathrm{T}}\right)$.

(5) All fixed points of $\alpha_{n}$ in $-1 \leq y<-|x|$ are $\alpha_{n}$-dual in pairs, as indicated above. This region, which is $\Pi_{z} \Pi\left\{\left(\theta_{1}, \theta_{2}\right): 0<\theta_{2}-\theta_{1}<1 / 2<\theta_{1}+\theta_{2}<1\right\}$, is one of the four components of $\Pi_{z} \Pi\left(\partial \mathcal{T} \backslash\left(\mathcal{T}_{+} \cup \mathcal{T}_{-}\right)\right)$and $\alpha_{n}$-duality is determined in the other three $|x|<y \leq 1,-1 \leq x<-|y|$ and $|y|<x \leq 1$ from these by symmetry. Together with $x= \pm y$ in $\$ \$ 2$ and 3 this accounts for all the fixed points in $\partial \mathcal{T}$.

Proof. A curve of fixed points in $\mathcal{T}$ joining two $\alpha_{n}$-dual fixed points on $\partial \mathcal{T}$ cannot, by Proposition 6.1(5), cross $x=\cos (\pi i / n), i$ odd, except where $y=0$ (nor $y=$ $\cos (\pi j / n), j$ odd, except where $x=0)$ because $z=x\left(1+U_{n-2}(y)\right) / U_{n-1}(y) \rightarrow \pm \infty$ as $(x, y)$ approaches a point $(\cos (\pi i / n), \cos (\pi j / n))$ with $i, j$ both odd along $J_{n}(x, y)=0$; see Proposition 2.6(x). Therefore, we study in $\mathbb{T}^{2}$ which pairs of reversing fixed points in $\partial \mathcal{T}$ are not separated by the planes $x=\cos (\pi i / n)$ or $y=\cos (\pi j / n), i, j$ odd. The preimages by $\Pi$ of these planes are the lines $\theta_{1}$ or $\theta_{2}=\left(k+\frac{1}{2}\right) / n, 0 \leq k<n$ in $\mathbb{T}^{2}$. (These lines have not been drawn in Figure 4 , and nor have their images by $\Pi_{z}$ in Figure 3.)

The $n^{2}$ preserving fixed points $(i / n, j / n)^{\mathrm{T}}, i, j \in\{0, \ldots, n-1\}$, by Lemma 3.6 , are at the centre of the squares into which these lines divide $\mathbb{T}^{2}$, one in each component. We shall exhibit one reversing point in many of these squares. In fact, of the $n^{2}-4=$ $\left|\operatorname{det}\left(I+\Phi\left(\alpha_{n}\right)\right)\right|$ reversing points (as in Lemma 3.6), there is exactly one in each of the $n^{2}$ squares with centre at a preserving point $(i / n, j / n)^{\mathrm{T}}, 0 \leq i, j<n$, because the others are accounted for as follows: (a) eight cases like $(1 / 4,1 / 2)^{\mathrm{T}}$ where there are none, (b) four cases like $(1 / 4,1 / 4)^{\mathrm{T}}$ where there are two, and (c) four cases like $(1 / 2,0)^{\mathrm{T}}$ where the reversing and preserving points are coincident; cases (a) give the points of $\partial \mathcal{T}$ on the $x$ and $y$-axes (twice each), cases (b) give the points on the $z$-axis (see Figure 1) and cases (c) give the vertices $V$ of $\mathcal{T}$. Where two preserving points are connected by a vertical line of fixed points (as in Lemma 2.12) the nearby reversing points are $\alpha_{n}$-dual, as in (1) and (2), or, in a different way, in (3). Otherwise the preserving point is $\alpha_{n}$-dual to a reversing point, as in (4).

(1) In $\mathbb{T}^{2}$ the reversing and preserving points

$$
(n / 4+1+k) v_{1}+(n / 4-k+j) v_{2} \text { and }(1 / 4+k / n, 1 / 2+j / n)^{\mathrm{T}}
$$

differ by

$$
\frac{1}{n^{2}-4}(-2 j+4 k / n-1, n / 2-2 k+4 j / n)^{\mathrm{T}} \text {. }
$$

In the range $k=1, \ldots, n / 4-2$ and $j=1, \ldots, n / 4-k-1$ the first coordinate is negative and is at least

$$
(-2(n / 4-2)+4 / n-1) /\left(n^{2}-4\right)=-\frac{1}{2 n}+(3+2 / n) /\left(n^{2}-4\right),
$$


so they are not separated by the line $\theta_{1}=1 / 4+\left(k-\frac{1}{2}\right) / n$. The second coordinate of the difference is positive and is at most $(n / 2-2+1-8 / n) /\left(n^{2}-4\right)$, which is less than $1 /(2 n)$ so the points are not separated by $\theta_{2}=1 / 2+\left(j+\frac{1}{2}\right) / n$.

Similarly, the two points

$$
(n / 4+1+k) v_{1}+(n / 4-k-j) v_{2} \text { and }(1 / 4+k / n, 1 / 2-j / n)^{\mathrm{T}}
$$

differ by

$$
\frac{1}{n^{2}-4}(2 j+4 k / n-1, n / 2-2 k-4 j / n)^{\mathrm{T}}
$$

and so they are not separated by the line $\theta_{1}=1 / 4+\left(k+\frac{1}{2}\right) / n$ nor by $\theta_{2}=1 / 2-\left(j-\frac{1}{2}\right) / n$. The preserving points $(1 / 4+k / n, 1 / 2 \pm j / n)$ are brought together by $\Pi_{z}$ at

$$
\Pi_{z}\left(\begin{array}{c}
1 / 4+k / n \\
1 / 2 \pm j / n
\end{array}\right)=\left(\begin{array}{c}
\cos (2 \pi(k+n / 4) / n) \\
-\cos (2 \pi j / n)
\end{array}\right)
$$

while the two reversing points $(n / 4+1+k) v_{1}+(n / 4-k \pm j) v_{2}$ have their image in the same one of the components into which $(x, y)$-space is divided by the lines $x=$ $\cos \left(2 \pi\left(k+n / 4 \pm \frac{1}{2}\right) / n\right)$ and $y=-\cos \left(2 \pi\left(j \pm \frac{1}{2}\right) / n\right)$. However, of the four components into which this component is divided by the lines with $x=\cos (2 \pi(k+n / 4) / n)$ and $y=\cos (2 \pi j / n)$, they lie in opposite ones. The two preserving points are $\alpha_{n}$-dual along the vertical line $(\cos (2 \pi(k+n / 4) / n),-\cos (2 \pi j / n), z)$ of fixed points, and so the two reversing points are also $\alpha_{n}$-dual along the curve $J_{n}=0$, which, by Proposition 6.1(4), must go through $(\cos (2 \pi(k+n / 4) / n),-\cos (2 \pi j / n))^{\mathrm{T}}$. Applying L'Hôpital as in the proof of Proposition 3.7 to $y$ shows that this curve of fixed points meets that vertical line on the surface $z=x y$. (By Lemma 2.8 this is the point on that straight line which is on the lowest level for the function $E$; it is also a bifurcation point of the dynamics.)

(2) This corresponds to (1) under $\left(\theta_{1}, \theta_{2}\right) \mapsto(1 / 2,1)-\left(\theta_{1}, \theta_{2}\right)$ and $(x, y, z) \mapsto$ $(-x, y,-z)$.

(3) This reversing point differs in $\mathbb{T}^{2}$ from the nearest preserving point by

$$
\left(\left(\begin{array}{l}
1 / 4 \\
1 / 2
\end{array}\right) \pm(j-1 / 2) v_{2}\right)-\left(\left(\begin{array}{c}
1 / 4 \\
1 / 2
\end{array}\right)\right) \pm\left(\left(\begin{array}{c}
0 \\
j / n
\end{array}\right)\right)= \pm \frac{1}{n^{2}-4}\left(\begin{array}{c}
1-2 j \\
4 j / n-n / 2
\end{array}\right)
$$

For $j \in\{1, \ldots, n / 4-1\}$, both coordinates are (in the + case) negative and greater than $-1 /(2 n)$ so the two points are not separated by any line $\theta_{1}, \theta_{2}=\left(k+\frac{1}{2}\right) / n$. Now

$$
\Pi_{z} \Pi\left(\left(\begin{array}{c}
1 / 4 \\
1 / 2
\end{array}\right) \pm(j-1 / 2) v_{2}\right)=\left(\begin{array}{c}
\cos \left(\pi / 2 \mp 2 \pi(2 j-1) /\left(n^{2}-4\right)\right) \\
-\cos \left(\pi n(2 j-1) /\left(n^{2}-4\right)\right)
\end{array}\right)
$$

so these two points have the same $y$-coordinate and their $x$-coordinates are separated by $x=0$; the curve of fixed points joining them crosses $x=0$ (along which $J_{n}=0$ ) at $(0, y, 0)$ for some $y$, by Corollary 2.2 , although, in contrast to cases (1) and (2), Proposition 6.1(4) does not say that this curve meets the vertical line of fixed points $(0,-\cos (2 \pi j / n), z)$. Figure 3 shows that the curve does not meet that vertical line.

(4) This reversing and preserving point differ in $\mathbb{T}^{2}$ by

$$
(n / 4+1+k) v_{1}+(n / 4-k) v_{2}-\left(\begin{array}{c}
1 / 4+k / n \\
1 / 2
\end{array}\right)=\frac{n-4 k}{n^{2}-4}\left(\begin{array}{c}
-1 / n \\
1 / 2
\end{array}\right) .
$$


For $k=1, \ldots, n / 4-1$ the scalar $(n-4 k) /\left(n^{2}-4\right)$ is positive and less than $1 / n$ so that

$$
\Pi\left((n / 4+1+k) v_{1}+(n / 4-k) v_{2}\right) \quad \text { and } \quad \Pi\left((1 / 4+k / n, 1 / 2)^{\mathrm{T}}\right)
$$

are not separated by

$$
x=\cos \left(2 \pi\left(1 / 4+\left(k \pm \frac{1}{2}\right) / n\right)\right) \quad \text { and } \quad y=\cos \left(2 \pi\left(1 / 2+\frac{1}{2} / n\right)\right)=-\cos (\pi / n),
$$

so they are $\alpha_{n}$-dual. The second statement corresponds to the first under $\left(\theta_{1}, \theta_{2}\right) \mapsto$ $(1 / 2,1)-\left(\theta_{1}, \theta_{2}\right)$ and $(x, y, z) \mapsto(-x, y,-z)$.

(5) In $-1 \leq y<-|x|$ we have considered $(n / 4-1) n / 2$ reversing points on $\partial \mathcal{T}$, $(n / 4-1)(n / 4-2)$ of them in (1), $(n / 4-1)(n / 4-2)$ in (2), 2(n/4-1) in (3) and $2(n / 4-1)$ in (4). The involutions $S(x, y, z)=(-x, y,-z)$ and $R(x, y, z)=(y, x, z)$ that commute with $\alpha_{n}$ (see Proposition 2.5) have compositions $R(x, y, z)=(y, x, z), R S R(x, y, z)=$ $(x,-y,-z)$ and $S R(x, y, z)=(-y, x,-z)$ that take $-1 \leq y<-|x|$ to $-1 \leq x<$ $-|y|,|x|<y \leq 1$ and $|y|<x \leq 1$, respectively, commuting with $\alpha_{n}$ and so determine $\alpha_{n}$-duality for $(n / 4-1) n / 2$ reversing points in each of these regions. Not counting the vertices there are $n-2$ reversing points in each of $x=y$ and $x=-y$ (see Figure 1) giving a total of $2 n(n / 4-1)+(2 n-4)=\left(n^{2}-8\right) / 2$ reversing fixed points in $\partial \mathcal{T} \backslash V$, which are each double-covered by one of the $n^{2}-4-4$ reversing points of $\mathbb{T}^{2}$ that do not give vertices. Thus all the reversing points have been included in our description. This concludes the proof of Theorem 6.2.

\section{REFERENCES}

[ABG] Y. Avishai, D. Berend and D. Glaubman. Minimum-dimension trace maps for substitution sequences. Phys. Rev. Lett. 72 (1994), 1842-1845.

[BGJ] M. Baake, U. Grimm and D. Joseph. Trace maps, invariants, and some of their applications. Internat. J. Modern Phys. B 7 (1993), 1527-1550.

[BR] M. Baake and J. A. G. Roberts. Reversing symmetry group of $G l(2, Z)$ and $P G l(2, Z)$ matrices with connections to cat maps and trace maps. J. Phys. A: Math. Gen. 30 (1997), 1549-1573.

[Bi] J. Birman. Braids, Links and Mapping Class Groups (Annals of Mathematics Studies, 82). Princeton University Press, Princeton, NJ, 1974

[BC] W. Bosma and J. Cannon. MAGMA (University of Sydney), 1994.

[C] M. Casdagli. Symbolic dynamics for the renormalization map of a quasiperiodic Schrödinger equation. Comm. Math. Phys. 107 (1986), 295-318.

[CB] A. J. Casson and S. A. Bleiler. Automorphisms of Surfaces after Nielsen and Thurston (London Mathematical Society Student Texts, 9). Cambridge University Press, Cambridge, 1988.

[E] D. B. A. Epstein. Almost all subgroups of a Lie group are free. J. Algebra 19 (1971), 261-262.

[FLP] Fathi, Laudenbach and Poénaru. Travaux de Thurston sur les surfaces. Astérisque 66-67 (1979).

[FK] R. Fricke and F. Klein. Vorlesungen uber die Theorie der automorphen Funktionen, I. Teubner, Leipzig, 1897. Reprint: Johnson Reprint Corp., New York, 1965.

[Go] W. M. Goldman. Topological components of spaces of representations. Invent. Math. 93 (1988), 557-607.

[Hu1] S. P. Humphries. An approach to automorphisms of free groups and braids via transvections. Math. Z. 209 (1992), 131-152.

[Hu2] S. P. Humphries. An action of subgroups of mapping class groups on polynomial algebras. Preprint, 2005.

[Ig] K. Iguchi. A class of new invariant surfaces under the trace maps for nary Fibonacci lattices. J. Math. Phys. 35 (1994), 1008-1019. 
[KH] A. Katok and B. Hasselblatt. Introduction to the Modern Theory of Dynamical Systems (with a supplement by A. Katok and L. Mendoza) (Encyclopedia of Mathematics and its Applications, 54). Cambridge University Press, Cambridge, 1995.

[LW] A. P. Liu and Z. X. Wen. Characterizations of the trace maps associated with invertible substitution. J. Wuhan Univ. Natur. Sci. Ed. 49 (2003), 289-292.

[LPW] Q. H. Liu, J. Peyrière and Z. Y. Wen. Periodic polynomial of trace maps. Bull. Sci. Math. doi: 10.1016/j.bulsci.2006.04.004

[Ma] W. Magnus. Rings of Fricke characters and automorphism groups of free groups. Math. Z. 170 (1980), 91-103.

[MKS] W. Magnus, A. Karrass and D. Solitar. Combinatorial Group Theory. Dover, New York, 1976.

[PM] J. Palis Jr. and W. de Melo. Geometric Theory of Dynamical Systems. An Introduction. Translated from Portuguese by A. K. Manning. Springer, Berlin, 1982.

[P] J. Peyrière. Trace maps. Beyond Quasicrystals (Les Houches, 1994). Springer, Berlin 1995, pp. $465-480$.

[PWW] J. Peyrière, Z. X. Wen and Z. Y. Wen. On the dynamic behaviours of the iterations of the trace map associated with substitutive sequences. Nonlinear Problems in Engineering and Science-Numerical and Analytical Approach (Beijing, 1991). Science Press, Beijing, 1992, pp. 259-266.

[Ri] T. J. Rivlin. The Chebyshev Polynomials. Wiley, New York, 1974.

[R] J. A. G. Roberts. Escaping orbits in trace maps. Phys. A 228 (1996), 295-325.

[RB1] J. A. G. Roberts and M. Baake. Trace maps as $3 D$ reversible dynamical systems with an invariant. J. Statist. Phys. 74 (1994), 829-888.

[RB2] J. A. G. Roberts and M. Baake. The dynamics of trace maps. Hamiltonian Mechanics (Toruń, 1993) (NATO Adv. Sci. Inst. Ser. B Phys., 331). Plenum, New York, 1994, pp. 275-285.

[Ro] C. Robinson. Dynamical Systems. Stability, Symbolic Dynamics, and Chaos, 2nd edn (Studies in Advanced Mathematics). CRC Press, Boca Raton, FL, 1999.

[WW] Z.-X. Wen and Z.-Y. Wen. A characterization of invertible trace maps associated with a substitution. Tokyo J. Math. 22 (1999), 65-74.

[W] R. F. Williams. The ' $D A$ ' maps of Smale and structural stability. Global Analysis (Proc. Symp. Pure Math., XIV, Berkeley, CA, 1968). American Mathematical Society, Providence, RI, 1970, pp. 329334. 